Original

\title{
Observation of Preputial Separation is a Useful Tool for Evaluating Endocrine Active Chemicals
}

\author{
Shinsuke Yoshimura ${ }^{1}$, Hajime Yamaguchi ${ }^{1}$, Kazunori Konno ${ }^{1}$, Noriko Ohsawa ${ }^{1}$, \\ Satoshi Noguchi ${ }^{1}$, and Akiko Chisaka ${ }^{1}$ \\ ${ }^{1}$ Hatano Research Institute, Food and Drug Safety Center, 729-5 Ochiai, Hadano, Kanagawa 257-8523, Japan
}

\begin{abstract}
Flutamide, $p, p^{\prime}$-dichlorodiphenyldichloroethylene, vinclozolin, diethylstilbestrol, ethynylestradiol and tamoxifen were administered by gavage to pregnant Sprague-Dawley rats on gestational days 14-17 or 18-21, and to male offspring on postnatal days $1-5,17-21$ or 35-39. The influence on the sexual maturation was assessed by preputial separation. Cleft phallus with hypospadias was induced by prenatal exposure to $10 \mathrm{mg} / \mathrm{kg}$ flutamide on gestational days 14-17 and 18-21, or administration of $100 \mathrm{mg} / \mathrm{kg}$ vinclozolin on gestational days $14-17$ to the dams. The day of preputial separation in these offspring could not be determined, because complete separation did not occur. Prenatal exposure of males to other chemicals did not affect the preputial separation. Postnatal exposure of $10 \mathrm{and} 30 \mathrm{mg} / \mathrm{kg}$ flutamide and $30 \mathrm{mg} / \mathrm{kg}$ vinclozolin led to delays of preputial separation. A marked delay was observed in males exposed to $100 \mu \mathrm{g} / \mathrm{kg}$ of ethynylestradiol or $3 \mathrm{mg} / \mathrm{kg}$ of tamoxifen on postnatal days 1-5. Diethylstilbestrol, $300 \mu \mathrm{g} / \mathrm{kg}$, administration on postnatal days 1-5 and 35-39 caused a delay in preputial separation. These results indicate that observing preputial separation is useful for evaluating anti-androgen treatment in the prepubertal period, and estrogenrelated chemical treatment from the neonatal period. (J Toxicol Pathol 2005; 18: 141-157)
\end{abstract}

Key words: preputial separation, rat, flutamide, vinclozolin, ethynylestradiol, diethylstilbestrol

\section{Introduction}

Preputial separation, which is the separation of the prepuce from the glans penis, is used as an indication of puberty in the male rat. Histological observations on the progress of preputial separation after cornification at the lining of the prepuce and surface of the glans penis were well described using Long-Evans rats in $1942^{1}$. We showed similar histological changes in Sprague-Dawley rats examined from postnatal day (PND) 6 to PND 56 $6^{2}$. Preputial separation is thought to be dependent on androgens, since castration blocks preputial separation and the addition of testosterone (TS) or dihydrotestosterone (DHT) nullifies the effect of castration ${ }^{1,3}$. In recent years, various in vivo screening assays have been developed for detecting endocrine disrupting chemicals. The uterotrophic assay is a method for detecting estrogenic or anti-estrogenic effects of chemicals on the weight of the uterus using immature or ovariectomized female rats. The Hershberger assay is a screening test to detect androgenic or anti-androgenic effects

Received: 10 March 2005, Accepted: 5 July 2005

Mailing address: Shinsuke Yoshimura, Laboratory of Toxicology, Hatano Research Institute, Food and Drug Safety Center, 729-5,

Ochiai, Hadano, Kanagawa 257-8523, Japan

TEL: 81-463-82-4751 FAX: 81-463-82-9627

E-mail: yoshimura.s@fdsc.or.jp of chemicals on the weight of castrated male reproductive organs such as the ventral prostate and seminal vesicles. The enhanced OECD Test Guideline 407 is a draft, new version of the Repeated Dose 28-day Oral Toxicity Study in Rodents, and is designed to detect the endocrine effect of chemicals. Also, the rodent 20-day thyroid/pubertal male assay has been proposed for evaluating chemicals influencing male puberty. In this assay, weaning male rats are continuously dosed by gavage beginning one week before puberty (which occurs at about PND 40) until PND 53 , and their puberty is measured by determining their age at preputial separation. Both estrogenic and anti-androgenic chemicals may induce delays in male puberty. Although the observation of preputial separation is a useful tool for detecting sexual maturation, the adequate administration period or relationship between dose and effect has not been sufficiently investigated. Accordingly, we performed a preliminary study to find out the suitable administration period or the most sensitive period using the following wellknown chemicals: flutamide, $p, p^{\prime}-$ dichlorodiphenyldichloroethylene, vinclozolin, diethylstilbestrol, ethynylestradiol and tamoxifen. Flutamide (FLU) ${ }^{4,5}$ is an anti-androgenic drug and is used in the Hershberger assay as the positive control agent. $p, p^{\prime}-$ dichlorodiphenyldichloroethylene (DDE) $)^{4-7}$ and vinclozolin $(\mathrm{VZ})^{7,8}$ are anti-androgenic chemicals and are used in the OECD validation study of the Hershberger assay to verify 
the effectiveness of this screening assay. Diethylstilbestrol (DES) is an estrogenic compound and has been used in various experimental studies. Ethynylestradiol (EE) is a synthetic estrogen and is used as a positive control in the uterotrophic assay, and tamoxifen (TAM) is used as a positive control for the anti-estrogenic effect in this screening assay.

To determine the administration period, the following reports were used as references. Induction of hypospadias has been reportedly caused by anti-androgens ${ }^{9}$. Puberty is undetermined in males with hypospadias because complete separation in the glans penis is not evident in these animals. A sensitive prenatal period for hypospadias is known to exist, thus, we selected gestational days (GD) 18-21 as the low sensitive period for prenatal exposure. We selected GD 14-17 as the high sensitive period for comparative study, but the high dose group of some chemicals may not be used for the observation of preputial separation. To examine the effects on the neonatal period, newborn rats were orally administered test chemicals on PND 1-5. Leydig cells of neonatal rats are known as fetal Leydig cells, and adult type Leydig cells appear from about PND $14^{10}$. It was reported that male rat serum testosterone levels reach a maximum on GD 19, decrease on PND 12, and then increase ${ }^{11}$. Thus, we also chose to administer the test chemicals during PND 1721 and PND 35-39 (prepubertal period). The 4 or 5 days of administration period in the present study is short compared to the Hershberger assay (10 days) or pubertal male assay (20 days), thus the effect on the animals is thought to be less than that seen in these assays. The purpose of this preliminary study was to find out the sensitive period for the screening assay, and more prolonged administration may show more pronounced effects on the animals. The number of animals in each group was determined referring to the other screening assays such as the uterotrophic assay (6 female rats in a group), the Hershberger assay (6 male rats in a group), and the Enhanced OECD Test Guideline 407 or pubertal male assay (10 male rats and 10 female rats). The number of pups and litters may be insufficient to determine the effects of unknown chemicals, but the purpose of the present study was to obtain the suitable period of administration for a screening study by the observation of preputial separation, thus we performed the preliminary study using the experimental design described in the following section.

\section{Materials and Methods}

\section{Animals and housing conditions}

Sprague-Dawley rats (Crj:CD (SD) IGS), 210 males and 421 females, 11 weeks of age, were obtained from Charles River Japan, Inc. (Atsugi, Japan). All animals were acclimatized to laboratory conditions and quarantined for about one week before mating. Rats used for this study were selected based upon their general appearance and behavior during the acclimatization period. Animals were housed individually in wire-bottom metal cages $(220 \times 270 \times 190$ $\mathrm{mm}$ ) and kept in a barrier sustained animal room that was maintained at $21.0-25.0^{\circ} \mathrm{C}$ and $40.0-75.0 \%$ relative humidity with a 12-hour artificial light cycle (lighting from 7:00 to 19:00). The room air was changed fifteen times per hour, and a commercial diet, CE-2 (CLEA Japan, Inc., Tokyo, Japan), and water (Hadano City) were available $a d$ libitum throughout the study. The Animal Use Committee of the Hatano Research Institute approved the study protocol.

To obtain pregnant animals, 12-week-old females were cohabited overnight on a 1:1 basis with males 12 weeks of age or older. Females were considered to be at GD 0 when daily examination revealed a vaginal plug. All pregnant animals were housed in cages with animal bedding, PAPER CLEAN $^{\circledR}$ (Japan SLC, Inc., Shizuoka, Japan), from GD 18 and allowed to give birth. The dams and pups were housed in wire-bottom metal cages after postpartum day 10.

For prenatal exposure, pregnant females were randomly assigned to groups consisting of 3 to 5 based on body weight before administration. For PND 1-5 exposure (PND 0 is the day of delivery), all female pups were discarded on PND 1 and the number of males per litter was adjusted to 5. The litters were then allocated to groups consisting of 3 or 4 litters (except for one group consisting 2 litters) based on pup mean body weight. On PND 6, the number of pups for prenatal or neonatal exposure and that for premature exposure was adjusted to 4 males per litter. For PND 17-21 and 35-39 exposure, litters were allocated to groups a few days before administration based on mean body weight. The numbers of litters or pups were decided referring to the other screening assays.

\section{Chemicals and treatment}

FLU, DES, EE and TAM were purchased from SigmaAldrich (St. Louis, MO), DDE was obtained from Aldrich Chemical (Milwaukee, WI), and VZ from Wako Pure Chemical (Osaka, Japan). Each chemical was dissolved in corn oil (Nacalai Tesque, Inc., Kyoto, Japan). Dosage levels for FLU were 1, 10 and $30 \mathrm{mg} / \mathrm{kg} /$ day, for DDE 10, 30, 100 and $300 \mathrm{mg} / \mathrm{kg} /$ day, for $\mathrm{VZ} \mathrm{10,30} \mathrm{and} 100 \mathrm{mg} / \mathrm{kg} / \mathrm{day}$, for DES $0.1,1,10,100$ and $300 \mu \mathrm{g} / \mathrm{kg} / \mathrm{day}$, for EE 10 and 100 $\mu \mathrm{g} / \mathrm{kg} / \mathrm{day}$, and for TAM 0.03 and $0.1 \mathrm{mg} / \mathrm{kg} /$ day (prenatal exposure) or $0.3,1$ and $3 \mathrm{mg} / \mathrm{kg} /$ day (postnatal exposure). The dosages employed in this study were based on the results from preliminary studies or those reported in the literature.

The chemicals were orally administered by gavage ( 5 or $10 \mathrm{~mL} / \mathrm{kg} \mathrm{BW}$ ) to pregnant female rats on GD 14-17 or 1821 , or to males on PND $1-5,17-21$ or $35-39$ (Fig. 1). In addition, EE was administered on PND 6-10 or $11-15$. The control animals were administered vehicle corn oil orally, at the same time periods and volume as the test group. An ATOM indwelling feeding tube (Atom Medical, Tokyo, Japan) was used for neonatal administration as described by Watanabe $^{12}$ and a stomach tube was used for adult and premature animals. 


\begin{tabular}{l} 
Mating \\
\cline { 2 - 4 } \\
\cline { 2 - 4 }
\end{tabular}

Fig. 1. Schedule of prenatal and postnatal treatment.

: period of chemical exposure, GD: gestational day, PND: postnatal day, AGD: measurement of anogenital distance on PND 6, PPS: observation of preputial separation from PND 35.

\section{Examination and measurement}

Body weights of pregnant females were measured on GD 0 , on the day of grouping before administration, and during the administration period. Body weights of males were measured on PND 0, 6, 22, 35, 56, the day of complete preputial separation, on the day of grouping, and during the administration period. Body weights on PND 0 to 6 were measured as mean value of each litter, and after PND 22 they were measured individually. The anogenital distance (AGD) of prenatally exposed male pups was measured on PND 6 before adjusting to 4 males per litter, with a digital micrometer (reproducible precision of $0.01 \mathrm{~mm}$, Digimatic caliper CD-15CP, Mitutoyo Corporation, Kanagawa, Japan). The progress of preputial separation in the males was observed macroscopically ${ }^{13}$ from PND 35. Surviving males were sacrificed under anesthesia on PND 56 and autopsy was performed. Male fatalities were autopsied as early as possible after they were discovered. Following the macroscopic examination, the testes, epididymides, ventral prostate, seminal vesicles, prepuce and penis were excised and fixed in $0.1 \mathrm{~mol} / \mathrm{L}$ phosphate buffered $10 \%$ formalin solution. Weights of the testes, epididymides, ventral prostate, and seminal vesicles of sacrificed animals were measured on the next day. Representative organs of 169 cases were embedded in paraffin, and the sections were then stained with hematoxylin-eosin (H\&E) for histopathological examination. To observe early stage hypospadias some of the discarded males were sacrificed on PND 6 and examined histologically as described above.

\section{Statistical analysis}

Body weights on the day of preputial separation and autopsy, the day of preputial separation, organ weights, AGD, and correction values of AGD [AGD (mm) $/ \sqrt[3]{\text { body }}$ $\overline{\text { weight }(\mathrm{g})}$ of pups] were statistically analyzed using the litter as the unit. Body weights on the day of preputial separation, the day of preputial separation, and organ weights in postnatally administered males were also analyzed using the individual values. These data were analyzed using Bartlett's test. When homogeneity of variance was confirmed, one- way analysis of variance was applied to detect significant differences among the groups. If a significant difference was detected among the groups, Dunnett's test was applied for multiple comparisons. When variance was not homogeneous, the Kruskal-Wallis analysis of ranks was applied. If a significant effect was detected among the groups, Dunnett's test was applied for multiple comparisons. The day of preputial separation and the body weights on the day of preputial separation between the two groups were analyzed by Student's or Welch's t-test. Comparisons between groups were made using $\mathrm{P} \leq 0.05$ as the level of significance. When preputial separation was not complete on the day of autopsy, the day of separation was set as day 56 for the analysis. The correlation between the day of preputial separation and body weight was analyzed using Pearson's correlation coefficient.

\section{Results}

\section{Prenatal exposure}

\section{1) Effects on the pregnant females}

In the groups exposed to $300 \mathrm{mg} / \mathrm{kg}$ of DDE on GD 1417 or GD $18-21$, body weights decreased during the administration period and either the pregnant females or their pups died. Depression of the weight gain was also observed in the $100 \mathrm{mg} / \mathrm{kg}$ DDE group exposed on GD 1821 , and all the pups in 3 litters out of 4 died. Deaths of pregnant females or pups were observed in the VZ, DES and TAM groups. Although depression of weight gain was observed in the EE group, all females survived and delivered pups. In the VZ, DES and TAM groups, one or two females delivered before the last day of administration period, and these females were excluded from the experiment.

\section{2) Malformations and inflammatory lesions of the genital organ}

The incidence of malformations and inflammatory lesions after prenatal exposure to chemicals are summarized in Table 1. On macroscopic examination, the glans penis of control males was covered with prepuce, and the prepuce could be completely retracted to expose the glans penis before PND 56 (Figs. 2A, 2B). The prepuce of males from dams exposed to $10 \mathrm{mg} / \mathrm{kg}$ of FLU on GD 14-17 had a cleft at the ventral aspect (cleft prepuce) and the glans penis was observed from the cleft. The ventral part of the penis was incompletely formed (cleft phallus) and the os penis was often exposed. The external urethral orifice of males with a cleft phallus opened at the ventral surface of the penis (hypospadias). Hypospadias was usually observed with cleft phallus. The incidence of a cleft prepuce was $25 \%$ ( 3 cases in 2 litters) and the incidence of a cleft phallus was 58\% (7 cases in all of the 3 litters), although a cleft prepuce was usually observed with a cleft phallus. Five males had no cleft on their prepuce or phallus. Although there was no cleft at the prepuce of males from dams exposed to FLU on GD 18-21, the ventral part of the penis was incompletely formed (cleft phallus); the incidence of the cleft phallus in this group was $25 \%$ ( 3 cases in one litter). 
Table 1. Malformation and Inflammatory Lesion in Genital Organ of Male Rats Prenatally Exposed to Chemicals

\begin{tabular}{|c|c|c|c|c|c|c|c|c|c|}
\hline Chemical & Dosing period & Group & Males & Litters & $\begin{array}{c}\text { Cleft } \\
\text { prepuce }\end{array}$ & $\begin{array}{c}\text { Cleft } \\
\text { phallus }\end{array}$ & $\begin{array}{c}\text { Ectopic } \\
\text { testis }\end{array}$ & $\begin{array}{l}\text { Hypoplasia } \\
\text { of prostate }\end{array}$ & $\begin{array}{l}\text { Prostatitis/ } \\
\text { Vesiculitis }\end{array}$ \\
\hline \multirow[t]{6}{*}{$\overline{\text { FLU }}$} & \multirow[t]{3}{*}{ GD 14-17 } & Control & 12 & 3 & 0 & 0 & 0 & 0 & 0 \\
\hline & & $1 \mathrm{mg} / \mathrm{kg}$ & 12 & 3 & 0 & 0 & 0 & 0 & 0 \\
\hline & & $10 \mathrm{mg} / \mathrm{kg}$ & 12 & 3 & $3(2)$ & $7(3)$ & $2(2)$ & 0 & 0 \\
\hline & \multirow[t]{3}{*}{ GD 18-21 } & Control & 12 & 3 & 0 & 0 & 0 & 0 & 0 \\
\hline & & $1 \mathrm{mg} / \mathrm{kg}$ & 12 & 3 & 0 & 0 & 0 & 0 & 0 \\
\hline & & $10 \mathrm{mg} / \mathrm{kg}$ & 12 & 3 & 0 & $3(1)$ & 0 & 0 & $7(3)$ \\
\hline \multirow[t]{8}{*}{$\overline{\mathrm{DDE}}$} & \multirow[t]{4}{*}{ GD 14-17 } & Control & 12 & 3 & 0 & 0 & 0 & 0 & 0 \\
\hline & & $10 \mathrm{mg} / \mathrm{kg}$ & 12 & 3 & 0 & 0 & 0 & 0 & 0 \\
\hline & & $30 \mathrm{mg} / \mathrm{kg}$ & 16 & 4 & 0 & 0 & 0 & 0 & 0 \\
\hline & & $100 \mathrm{mg} / \mathrm{kg}$ & 16 & 4 & 0 & 0 & 0 & 0 & 0 \\
\hline & \multirow[t]{4}{*}{ GD $18-21$} & Control & 14 & 4 & 0 & 0 & 0 & 0 & 0 \\
\hline & & $10 \mathrm{mg} / \mathrm{kg}$ & 16 & 4 & 0 & 0 & 0 & 0 & 0 \\
\hline & & $30 \mathrm{mg} / \mathrm{kg}$ & 14 & 4 & 0 & 0 & 0 & 0 & 0 \\
\hline & & $100 \mathrm{mg} / \mathrm{kg}$ & 4 & 1 & 0 & 0 & 0 & 0 & 0 \\
\hline \multirow[t]{8}{*}{$\overline{V Z}$} & \multirow[t]{4}{*}{ GD 14-17 } & Control & 20 & 5 & 0 & 0 & 0 & 0 & 0 \\
\hline & & $10 \mathrm{mg} / \mathrm{kg}$ & 20 & 5 & 0 & 0 & 0 & 0 & 0 \\
\hline & & $30 \mathrm{mg} / \mathrm{kg}$ & 20 & 5 & 0 & 0 & 0 & 0 & 0 \\
\hline & & $100 \mathrm{mg} / \mathrm{kg}$ & 20 & 5 & $17(5)$ & $17(5)$ & $1(1)$ & $1(1)$ & 0 \\
\hline & \multirow[t]{4}{*}{ GD 18-21 } & Control & 20 & 5 & 0 & 0 & 0 & 0 & 0 \\
\hline & & $10 \mathrm{mg} / \mathrm{kg}$ & 16 & 4 & 0 & 0 & $1(1)$ & 0 & 0 \\
\hline & & $30 \mathrm{mg} / \mathrm{kg}$ & 16 & 4 & 0 & 0 & 0 & 0 & 0 \\
\hline & & $100 \mathrm{mg} / \mathrm{kg}$ & 15 & 4 & 0 & 0 & 0 & 0 & 0 \\
\hline \multirow[t]{12}{*}{ DES } & \multirow[t]{6}{*}{ GD 14-17 } & Control & 19 & 5 & 0 & 0 & 0 & 0 & 0 \\
\hline & & $0.1 \mu \mathrm{g} / \mathrm{kg}$ & 12 & 3 & 0 & 0 & 0 & 0 & 0 \\
\hline & & $1 \mu \mathrm{g} / \mathrm{kg}$ & 20 & 5 & 0 & 0 & 0 & 0 & 0 \\
\hline & & $10 \mu \mathrm{g} / \mathrm{kg}$ & 16 & 4 & 0 & 0 & 0 & 0 & 0 \\
\hline & & $100 \mu \mathrm{g} / \mathrm{kg}$ & 16 & 4 & 0 & 0 & 0 & 0 & 0 \\
\hline & & $300 \mu \mathrm{g} / \mathrm{kg}$ & 12 & 4 & 0 & 0 & 0 & 0 & 0 \\
\hline & \multirow[t]{6}{*}{ GD 18-21 } & Control & 16 & 4 & 0 & 0 & 0 & 0 & 0 \\
\hline & & $0.1 \mu \mathrm{g} / \mathrm{kg}$ & 20 & 5 & 0 & 0 & 0 & 0 & 0 \\
\hline & & $1 \mu \mathrm{g} / \mathrm{kg}$ & 16 & 4 & 0 & 0 & 0 & 0 & 0 \\
\hline & & $10 \mu \mathrm{g} / \mathrm{kg}$ & 20 & 5 & 0 & 0 & 0 & 0 & 0 \\
\hline & & $100 \mu \mathrm{g} / \mathrm{kg}$ & 20 & 5 & 0 & 0 & 0 & 0 & 0 \\
\hline & & $300 \mu \mathrm{g} / \mathrm{kg}$ & 7 & 2 & 0 & 0 & 0 & $1(1)$ & 0 \\
\hline \multirow[t]{6}{*}{$\overline{\mathrm{EE}}$} & \multirow[t]{3}{*}{ GD 14-17 } & Control & 16 & 4 & 0 & 0 & 0 & 0 & 0 \\
\hline & & $10 \mu \mathrm{g} / \mathrm{kg}$ & 16 & 4 & 0 & 0 & 0 & 0 & 0 \\
\hline & & $100 \mu \mathrm{g} / \mathrm{kg}$ & 20 & 5 & 0 & 0 & 0 & 0 & 0 \\
\hline & \multirow[t]{3}{*}{ GD 18-21 } & Control & 19 & 5 & 0 & 0 & 0 & 0 & 0 \\
\hline & & $10 \mu \mathrm{g} / \mathrm{kg}$ & 15 & 4 & 0 & 0 & 0 & 0 & 0 \\
\hline & & $100 \mu \mathrm{g} / \mathrm{kg}$ & 16 & 4 & 0 & 0 & 0 & 0 & 0 \\
\hline \multirow[t]{6}{*}{ TAM } & \multirow[t]{3}{*}{ GD $14-17$} & Control & 16 & 4 & 0 & 0 & 0 & 0 & 0 \\
\hline & & $0.03 \mathrm{mg} / \mathrm{kg}$ & 15 & 4 & 0 & 0 & 0 & 0 & 0 \\
\hline & & $0.1 \mathrm{mg} / \mathrm{kg}$ & 7 & 2 & 0 & 0 & 0 & 0 & 0 \\
\hline & \multirow[t]{3}{*}{ GD 18-21 } & Control & 19 & 5 & 0 & 0 & 0 & 0 & 0 \\
\hline & & $0.03 \mathrm{mg} / \mathrm{kg}$ & 12 & 3 & 0 & 0 & 0 & 0 & 0 \\
\hline & & $0.1 \mathrm{mg} / \mathrm{kg}$ & 11 & 3 & 0 & 0 & 0 & 0 & 0 \\
\hline
\end{tabular}

Value: number of cases (litters) with abnormality.

FLU: flutamide; DDE: $p, p^{\prime}$-dichlorodiphenyldichloroethylene; VZ: vinclozolin; DES: diethylstilbestrol; EE: ethynylestradiol; TAM: tamoxifen; GD: gestational day.

Cleft prepuce and cleft phallus were also observed in males from dams exposed to $100 \mathrm{mg} / \mathrm{kg}$ of VZ on GD 14-17 (Figs. 2C, 2D). The incidence of cleft prepuce and cleft phallus was $85 \%$ ( 17 cases in all of the 5 litters). However, there was no cleft at the prepuce or phallus of males from dams exposed to VZ on GD 18-21 (Figs. 2E, 2F). The time of sexual maturation is determined by complete separation of the prepuce from the ventral surface of the glans penis, but preputial separation could not determined in males with cleft phallus, since complete separation in the glans penis was not evident.

The testis of males from dams exposed to $10 \mathrm{mg} / \mathrm{kg}$ of 


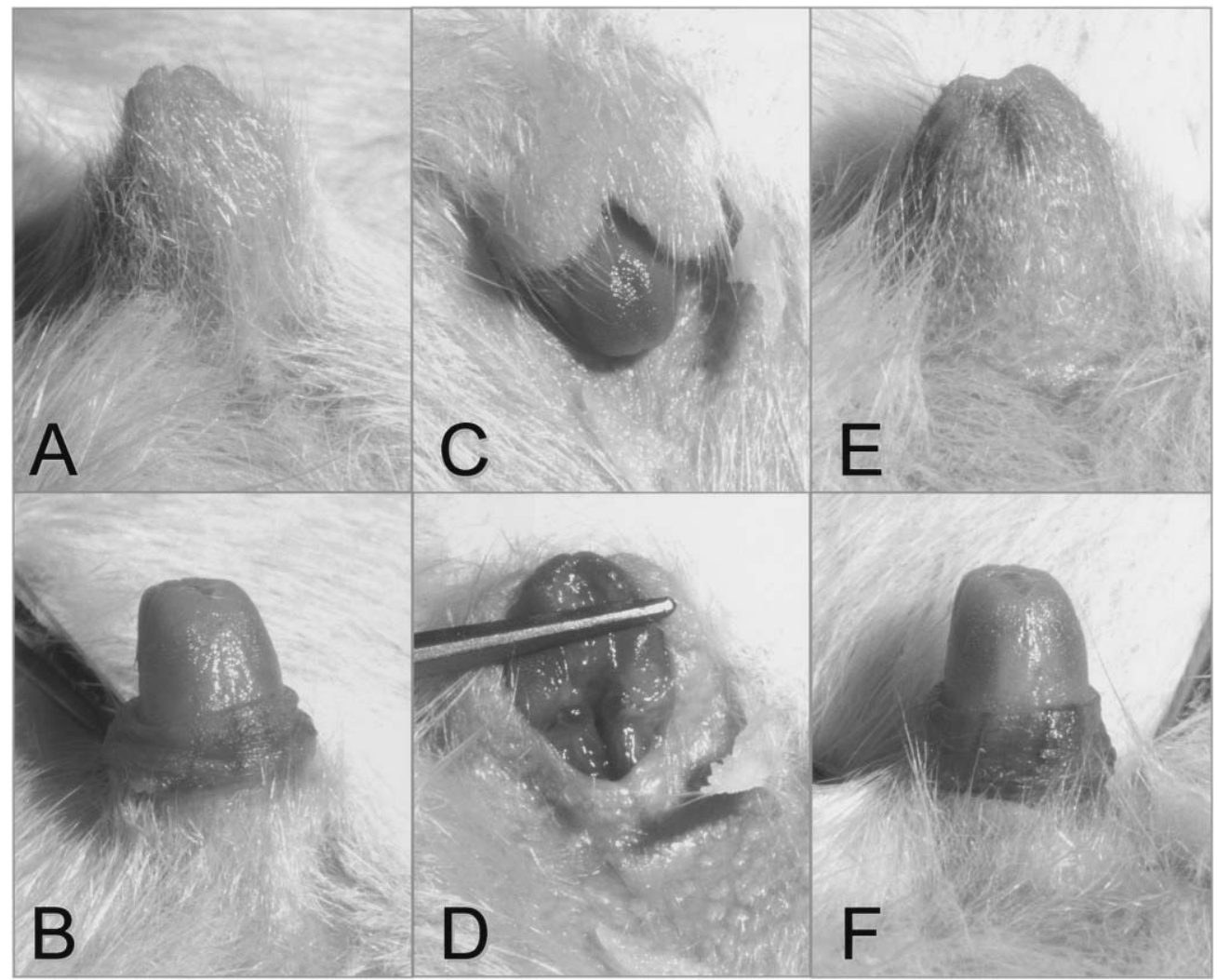

Fig. 2. External genitalia of male rats on PND 56.

$\mathrm{A}$ and B: Control male rat. Prepuce is completely retracted. C and D: Male rat from a dam exposed to $100 \mathrm{mg} / \mathrm{kg}$ vinclozolin on GD 14-17. Ventral side of the prepuce has a cleft and the glans penis is observed from the cleft (C). The ventral part of the penis is incompletely formed (cleft phallus, shown in D). E and F: Male rat from a dam exposed to $100 \mathrm{mg} / \mathrm{kg}$ vinclozolin on GD 18-21. There was no cleft on the ventral surface of the prepuce or penis.

FLU on GD 14-17 (17\%, 2 cases in 2 litters) or $100 \mathrm{mg} / \mathrm{kg}$ of $\mathrm{VZ}$ on GD $14-17(5 \%$, one case in one litter) did not descend into the scrotum and was located in the ventral subcutis (ectopic testis) instead. One male from a dam exposed to $10 \mathrm{mg} / \mathrm{kg} \mathrm{VZ}$ on GD 18-21 also had an ectopic testis. Ectopic testis was observed unilaterally. Marked inflammation in the prostate and seminal vesicle occurred in the group exposed to $10 \mathrm{mg} / \mathrm{kg}$ FLU on GD 18-21, and 5 of 12 males in this group died from the severe inflammation. Males with marked prostatitis and vesiculitis did not show hypospadias in their glans penis.

\section{3) Preputial separation}

Males with hypospadias were excluded from the preputial separation analysis, and the resulting days of preputial separation of males without hypospadias are summarized in Table 2. Preputial separation in males from dams exposed to $10 \mathrm{mg} / \mathrm{kg}$ of FLU was significantly delayed in both GD 14-17 and GD 18-21 treatment groups, and 2 males in the GD 18-21 treatment group had incomplete preputial separation on the day of autopsy, PND 56. In the group exposed to $100 \mathrm{mg} / \mathrm{kg}$ of DDE on GD 18-21, all pups of 3 litters died. There were no differences among the other 3 groups. In the $100 \mathrm{mg} / \mathrm{kg} \mathrm{VZ}$ groups, male pups without hypospadias showed no difference from the control group, and the lower dose groups did not show any significant difference from the control. Two of 4 pregnant females administered $300 \mu \mathrm{g} / \mathrm{kg}$ of DES from GD 18 were excluded from the experiment because these females delivered their pups on GD 21 before the end of the administration period. Preputial separation of male offspring exposed to DES was not different from the control group. EE and TAM administered in any exposure period did not affect preputial separation.

Body weight on the day of complete preputial separation was significantly higher in males from dams exposed to FLU on GD 18-21, but there were no changes in other chemically-treated groups.

\section{4) Measurement of AGD on PND 6}

Table 3 shows the AGD, body weight, and correction

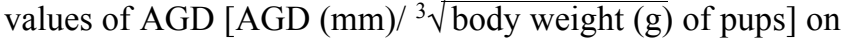
PND 6. Correction values of AGD were significantly decreased in the groups exposed to $10 \mathrm{mg} / \mathrm{kg}$ FLU on GD 14-17 or GD 18-21, and the group exposed to $100 \mathrm{mg} / \mathrm{kg}$ $\mathrm{VZ}$ on GD 14-17. DES induced a significant reduction in AGD of the 100 and $300 \mu \mathrm{g} / \mathrm{kg}$ groups exposed on GD 1821. DDE, EE and TAM did not induce a significant 
Table 2. Preputial Separation and Body Weights of Male Rats Prenatally Exposed to Chemicals

\begin{tabular}{|c|c|c|c|c|c|c|c|c|c|c|c|c|}
\hline \multirow{2}{*}{$\begin{array}{l}\text { Chemical } \\
\text { FLU }\end{array}$} & \multirow{2}{*}{$\begin{array}{l}\begin{array}{l}\text { Dosing } \\
\text { period }\end{array} \\
\text { GD 14-17 }\end{array}$} & \multirow{2}{*}{$\frac{\text { Group }}{\text { Control }}$} & \multirow{2}{*}{$\begin{array}{c}\text { Males } \\
12\end{array}$} & \multirow{2}{*}{$\frac{\text { Litters }}{3}$} & \multicolumn{4}{|c|}{$\begin{array}{l}\text { PND of preputial } \\
\text { separation }\end{array}$} & \multicolumn{3}{|c|}{$\begin{array}{c}\text { BW }(\mathrm{g}) \text { on } \\
\text { the day of PPS }\end{array}$} & \multirow{2}{*}{$\begin{array}{c}\begin{array}{c}\text { Incomplete } \\
\text { separation }\end{array} \\
0\end{array}$} \\
\hline & & & & & 42.8 & \pm & 2.2 & & 232.2 & \pm 26.5 & & \\
\hline & & $1 \mathrm{mg} / \mathrm{kg}$ & 12 & 3 & 42.4 & \pm & 0.3 & & 216.5 & $\pm \quad 3.3$ & & 0 \\
\hline & & $10 \mathrm{mg} / \mathrm{kg}$ & 5 & 2 & 49.1 & \pm & 0.6 & $* *$ & 271.2 & \pm 41.3 & & 0 \\
\hline & GD 18-21 & Control & 12 & 3 & 43.2 & \pm & 1.4 & & 227.0 & \pm 19.2 & & 0 \\
\hline & & $1 \mathrm{mg} / \mathrm{kg}$ & 12 & 3 & 44.5 & \pm & 0.7 & & 249.8 & \pm 12.7 & & 0 \\
\hline & & $10 \mathrm{mg} / \mathrm{kg}$ & 5 & 2 & 51.3 & \pm & 0.5 & $* *$ & 322.2 & $\begin{array}{r} \pm \quad 13.7 \\
\end{array}$ & $* *$ & $2(1)$ \\
\hline \multirow[t]{8}{*}{$\overline{\mathrm{DDE}}$} & GD $14-17$ & Control & 12 & 3 & 42.8 & \pm & 2.2 & & 232.2 & \pm 26.5 & & 0 \\
\hline & & $10 \mathrm{mg} / \mathrm{kg}$ & 12 & 3 & 42.6 & \pm & 0.4 & & 242.1 & \pm 23.3 & & 0 \\
\hline & & $30 \mathrm{mg} / \mathrm{kg}$ & 16 & 4 & 43.1 & \pm & 1.0 & & 248.3 & \pm 20.1 & & 0 \\
\hline & & $100 \mathrm{mg} / \mathrm{kg}$ & 16 & 4 & 44.6 & \pm & 3.0 & & 242.5 & $\pm \quad 14.0$ & & 0 \\
\hline & GD 18-21 & Control & 14 & 4 & 43.8 & \pm & 1.5 & & 231.7 & \pm 26.7 & & 0 \\
\hline & & $10 \mathrm{mg} / \mathrm{kg}$ & 16 & 4 & 43.3 & \pm & 1.6 & & 233.7 & \pm 19.6 & & 0 \\
\hline & & $30 \mathrm{mg} / \mathrm{kg}$ & 14 & 4 & 43.0 & \pm & 1.0 & & 239.3 & \pm 16.2 & & 0 \\
\hline & & $100 \mathrm{mg} / \mathrm{kg}$ & 4 & 1 & 43.5 & & & & 233.5 & & & 0 \\
\hline \multirow[t]{8}{*}{$\overline{V Z}$} & GD $14-17$ & Control & 20 & 5 & 44.4 & \pm & 1.2 & & 245.3 & \pm 10.5 & & 0 \\
\hline & & $10 \mathrm{mg} / \mathrm{kg}$ & 20 & 5 & 43.7 & \pm & 1.6 & & 243.7 & \pm 16.4 & & 0 \\
\hline & & $30 \mathrm{mg} / \mathrm{kg}$ & 20 & 5 & 44.5 & \pm & 1.7 & & 246.2 & \pm 13.7 & & 0 \\
\hline & & $100 \mathrm{mg} / \mathrm{kg}$ & 3 & 1 & 43.7 & & & & 254.1 & & & 0 \\
\hline & GD 18-21 & Control & 20 & 5 & 44.1 & \pm & 0.3 & & 252.1 & \pm 36.7 & & 0 \\
\hline & & $10 \mathrm{mg} / \mathrm{kg}$ & 16 & 4 & 43.8 & \pm & 1.5 & & 248.2 & $\pm \quad 17.3$ & & 0 \\
\hline & & $30 \mathrm{mg} / \mathrm{kg}$ & 16 & 4 & 43.3 & \pm & 1.9 & & 240.8 & $\pm \quad 11.4$ & & 0 \\
\hline & & $100 \mathrm{mg} / \mathrm{kg}$ & 15 & 4 & 45.1 & \pm & 1.7 & & 259.2 & $\begin{array}{l} \pm 22.3 \\
\end{array}$ & & 0 \\
\hline \multirow[t]{12}{*}{ DES } & GD $14-17$ & Control & 19 & 5 & 44.4 & \pm & 1.7 & & 246.4 & $\pm \quad 12.9$ & & 0 \\
\hline & & $0.1 \mu \mathrm{g} / \mathrm{kg}$ & 12 & 3 & 43.3 & \pm & 0.5 & & 242.3 & $\pm \quad 12.7$ & & 0 \\
\hline & & $1 \mu \mathrm{g} / \mathrm{kg}$ & 20 & 5 & 43.3 & \pm & 1.3 & & 243.3 & \pm 17.6 & & 0 \\
\hline & & $10 \mu \mathrm{g} / \mathrm{kg}$ & 16 & 4 & 42.8 & \pm & 1.3 & & 244.2 & $\pm \quad 19.9$ & & 0 \\
\hline & & $100 \mu \mathrm{g} / \mathrm{kg}$ & 16 & 4 & 43.7 & \pm & 1.4 & & 257.9 & $\pm \quad 8.4$ & & 0 \\
\hline & & $300 \mu \mathrm{g} / \mathrm{kg}$ & 12 & 4 & 46.1 & \pm & 1.0 & & 260.8 & \pm 20.5 & & 0 \\
\hline & GD 18-21 & Control & 16 & 4 & 44.6 & \pm & 1.1 & & 246.5 & \pm 16.0 & & 0 \\
\hline & & $0.1 \mu \mathrm{g} / \mathrm{kg}$ & 20 & 5 & 45.1 & \pm & 1.1 & & 255.9 & $\pm \quad 22.9$ & & 0 \\
\hline & & $1 \mu \mathrm{g} / \mathrm{kg}$ & 16 & 4 & 43.3 & \pm & 1.2 & & 252.5 & $\pm \quad 18.5$ & & 0 \\
\hline & & $10 \mu \mathrm{g} / \mathrm{kg}$ & 20 & 5 & 43.4 & \pm & 1.4 & & 241.6 & $\pm \quad 7.5$ & & 0 \\
\hline & & $100 \mu \mathrm{g} / \mathrm{kg}$ & 20 & 5 & 44.0 & \pm & 0.6 & & 245.3 & $\pm \quad 9.1$ & & 0 \\
\hline & & $300 \mu \mathrm{g} / \mathrm{kg}$ & 7 & 2 & 46.8 & \pm & 1.1 & & 239.9 & $\pm \quad 1.7$ & & 0 \\
\hline \multirow[t]{6}{*}{$\overline{\mathrm{EE}}$} & GD $14-17$ & Control & 16 & 4 & 44.4 & \pm & 1.0 & & 254.6 & $\pm \quad 11.9$ & & 0 \\
\hline & & $10 \mu \mathrm{g} / \mathrm{kg}$ & 16 & 4 & 45.6 & \pm & 2.0 & & 240.2 & $\begin{array}{l}11.2 \\
\pm \quad 6.2\end{array}$ & & 0 \\
\hline & & $100 \mu \mathrm{g} / \mathrm{kg}$ & 20 & 5 & 45.8 & \pm & 2.2 & & 261.6 & $\pm \quad 20.2$ & & 0 \\
\hline & GD 18-21 & Control & 19 & 5 & 43.6 & \pm & 1.7 & & 246.6 & $\begin{array}{ll} \pm \quad 9.6\end{array}$ & & 0 \\
\hline & & $10 \mu \mathrm{g} / \mathrm{kg}$ & 15 & 4 & 43.3 & \pm & 0.5 & & 248.3 & $\pm \quad 10.1$ & & 0 \\
\hline & & $100 \mu \mathrm{g} / \mathrm{kg}$ & 16 & 4 & 42.8 & \pm & 1.6 & & 247.6 & $\pm \quad 12.3$ & & 0 \\
\hline \multirow[t]{6}{*}{ TAM } & GD $14-17$ & Control & 16 & 4 & 44.4 & \pm & 1.0 & & 254.6 & $\pm \quad 11.9$ & & 0 \\
\hline & & $0.03 \mathrm{mg} / \mathrm{kg}$ & 15 & 4 & 44.5 & \pm & 1.3 & & 252.7 & $\pm \quad 25.2$ & & 0 \\
\hline & & $0.1 \mathrm{mg} / \mathrm{kg}$ & 7 & 2 & 43.5 & \pm & 0.3 & & 244.2 & $\pm \quad 11.6$ & & 0 \\
\hline & $\overline{\text { GD } 18-21}$ & Control & 19 & 5 & 43.6 & \pm & 1.7 & & 246.6 & $\pm \quad 9.6$ & & 0 \\
\hline & & $0.03 \mathrm{mg} / \mathrm{kg}$ & 12 & 3 & 43.2 & \pm & 1.0 & & 242.7 & $\pm \quad 32.3$ & & 0 \\
\hline & & $0.1 \mathrm{mg} / \mathrm{kg}$ & 11 & 3 & 43.7 & \pm & 0.6 & & 257.5 & $\pm \quad 30.3$ & & 0 \\
\hline
\end{tabular}

Value: Mean \pm S.D. calculated using the litter as the unit.

FLU: flutamide; DDE: $p, p^{\prime}$-dichlorodiphenyldichloroethylene; VZ: vinclozolin, DES: diethylstilbestrol; EE: ethynylestradiol; TAM: tamoxifen; GD: gestational day; PND: postnatal day; BW: body weight; PPS: preputial separation.

Incomplete separation: number of animals (litters) with incomplete separation on PND 56.

**: significantly different from control, $\mathrm{p}<0.01$. 
Table 3. Anogenital Distance (AGD) of PND 6 Male Rats Prenatally Exposed to Chemicals

\begin{tabular}{|c|c|c|c|c|c|c|c|c|c|c|c|c|c|c|c|}
\hline \multirow{2}{*}{$\begin{array}{l}\text { Chemical } \\
\text { FLU }\end{array}$} & \multirow{2}{*}{$\begin{array}{c}\begin{array}{c}\text { Dosing } \\
\text { period }\end{array} \\
\text { GD 14-17 }\end{array}$} & \multirow{4}{*}{$\begin{array}{l}\text { Group } \\
\text { Control } \\
1 \mathrm{mg} / \mathrm{kg} \\
10 \mathrm{mg} / \mathrm{kg}\end{array}$} & \multirow{2}{*}{$\frac{\text { Males }}{12}$} & \multirow{2}{*}{$\begin{array}{c}\text { Litters } \\
3\end{array}$} & \multicolumn{4}{|c|}{$\operatorname{AGD}(\mathrm{mm})$} & \multicolumn{4}{|c|}{$\begin{array}{c}\text { Correction value of } A G D \\
(\mathrm{~mm} / \sqrt[3]{\mathrm{g}})\end{array}$} & \multicolumn{3}{|c|}{ Body weight $(\mathrm{g})$} \\
\hline & & & & & 7.07 & \pm & 0.59 & & 2.85 & \pm & 0.11 & & 15.4 & \pm & 3.1 \\
\hline & & & 20 & 3 & 6.21 & \pm & 0.09 & & 2.63 & \pm & 0.03 & & 13.3 & \pm & 1.0 \\
\hline & & & 26 & 3 & 5.53 & \pm & 0.39 & $* *$ & 2.35 & \pm & 0.12 & $* *$ & 13.0 & \pm & 1.0 \\
\hline & GD 18-21 & Control & 19 & 3 & 6.70 & \pm & 0.25 & & 2.93 & \pm & 0.07 & & 11.9 & \pm & 0.7 \\
\hline & & $1 \mathrm{mg} / \mathrm{kg}$ & 20 & 3 & 7.04 & \pm & 0.71 & & 2.89 & \pm & 0.21 & & 14.4 & \pm & 1.3 \\
\hline & & $10 \mathrm{mg} / \mathrm{kg}$ & 22 & 3 & 5.82 & \pm & 0.45 & & 2.42 & \pm & 0.22 & $*$ & 14.1 & \pm & 1.2 \\
\hline \multirow[t]{8}{*}{$\overline{\mathrm{DDE}}$} & GD 14-17 & Control & 12 & 3 & 7.07 & \pm & 0.59 & & 2.85 & \pm & 0.11 & & 15.4 & \pm & 3.1 \\
\hline & & $10 \mathrm{mg} / \mathrm{kg}$ & 23 & 3 & 6.84 & \pm & 0.38 & & 2.84 & \pm & 0.01 & & 14.0 & \pm & 2.2 \\
\hline & & $30 \mathrm{mg} / \mathrm{kg}$ & 30 & 4 & 6.67 & \pm & 0.59 & & 2.78 & \pm & 0.13 & & 13.8 & \pm & 2.3 \\
\hline & & $100 \mathrm{mg} / \mathrm{kg}$ & 23 & 4 & 6.32 & \pm & 0.44 & & 2.71 & \pm & 0.11 & & 12.8 & \pm & 1.6 \\
\hline & GD 18-21 & Control & 19 & 4 & 7.55 & \pm & 0.86 & & 3.10 & \pm & 0.20 & & 14.5 & \pm & 2.3 \\
\hline & & $10 \mathrm{mg} / \mathrm{kg}$ & 26 & 4 & 6.96 & \pm & 0.56 & & 2.93 & \pm & 0.20 & & 13.4 & \pm & 1.1 \\
\hline & & $30 \mathrm{mg} / \mathrm{kg}$ & 26 & 4 & 7.29 & \pm & 0.45 & & 3.00 & \pm & 0.09 & & 14.5 & \pm & 2.9 \\
\hline & & $100 \mathrm{mg} / \mathrm{kg}$ & 5 & 1 & 7.40 & & & & 3.04 & & & & 14.5 & & \\
\hline \multirow[t]{8}{*}{$\overline{\mathrm{VZ}}$} & GD $14-17$ & Control & 35 & 5 & 6.75 & \pm & 0.39 & & 2.86 & \pm & 0.14 & & 13.2 & \pm & 0.9 \\
\hline & & $10 \mathrm{mg} / \mathrm{kg}$ & 38 & 5 & 6.60 & \pm & 0.14 & & 2.72 & \pm & 0.08 & & 14.5 & \pm & 1.9 \\
\hline & & $30 \mathrm{mg} / \mathrm{kg}$ & 28 & 5 & 6.46 & \pm & 0.22 & & 2.73 & \pm & 0.08 & & 13.2 & \pm & 0.4 \\
\hline & & $100 \mathrm{mg} / \mathrm{kg}$ & 34 & 5 & 4.87 & \pm & 0.48 & $* *$ & 2.02 & \pm & 0.22 & $* *$ & 14.1 & \pm & 2.2 \\
\hline & GD 18-21 & Control & 43 & 5 & 6.25 & \pm & 0.75 & & 2.65 & \pm & 0.29 & & 13.2 & \pm & 2.0 \\
\hline & & $10 \mathrm{mg} / \mathrm{kg}$ & 28 & 4 & 6.14 & \pm & 0.38 & & 2.59 & \pm & 0.15 & & 13.3 & \pm & 0.8 \\
\hline & & $30 \mathrm{mg} / \mathrm{kg}$ & 24 & 4 & 6.59 & \pm & 0.31 & & 2.70 & \pm & 0.06 & & 14.7 & \pm & 1.8 \\
\hline & & $100 \mathrm{mg} / \mathrm{kg}$ & 26 & 4 & 5.74 & \pm & 0.54 & & 2.41 & \pm & 0.20 & & 13.6 & \pm & 1.4 \\
\hline \multirow[t]{12}{*}{$\overline{\mathrm{DES}}$} & GD $14-17$ & Control & 36 & 5 & 6.25 & \pm & 0.41 & & 2.66 & \pm & 0.06 & & 13.0 & \pm & 1.9 \\
\hline & & $0.1 \mu \mathrm{g} / \mathrm{kg}$ & 20 & 3 & 6.35 & \pm & 0.13 & & 2.60 & \pm & 0.09 & & 14.6 & \pm & 0.8 \\
\hline & & $1 \mu \mathrm{g} / \mathrm{kg}$ & 45 & 5 & 6.35 & \pm & 0.20 & & 2.66 & \pm & 0.06 & & 13.8 & \pm & 1.7 \\
\hline & & $10 \mu \mathrm{g} / \mathrm{kg}$ & 21 & 4 & 6.64 & \pm & 0.54 & & 2.75 & \pm & 0.14 & & 14.1 & \pm & 1.8 \\
\hline & & $100 \mu \mathrm{g} / \mathrm{kg}$ & 26 & 4 & 6.40 & \pm & 0.62 & & 2.63 & \pm & 0.19 & & 14.4 & \pm & 1.3 \\
\hline & & $300 \mu \mathrm{g} / \mathrm{kg}$ & 17 & 4 & 5.77 & \pm & 0.83 & & 2.54 & \pm & 0.30 & & 11.6 & \pm & 0.9 \\
\hline & GD 18-21 & Control & 26 & 4 & 6.35 & \pm & 0.46 & & 2.68 & \pm & 0.15 & & 13.3 & \pm & 0.9 \\
\hline & & $0.1 \mu \mathrm{g} / \mathrm{kg}$ & 44 & 5 & 6.22 & \pm & 0.26 & & 2.64 & \pm & 0.09 & & 13.2 & \pm & 1.4 \\
\hline & & $1 \mu \mathrm{g} / \mathrm{kg}$ & 35 & 4 & 6.28 & \pm & 0.28 & & 2.63 & \pm & 0.06 & & 13.7 & \pm & 2.2 \\
\hline & & $10 \mu \mathrm{g} / \mathrm{kg}$ & 36 & 5 & 6.17 & \pm & 0.31 & & 2.62 & \pm & 0.09 & & 13.1 & \pm & 0.9 \\
\hline & & $100 \mu \mathrm{g} / \mathrm{kg}$ & 27 & 5 & 5.47 & \pm & 0.44 & $* *$ & 2.38 & \pm & 0.10 & $* *$ & 12.2 & \pm & 1.6 \\
\hline & & $300 \mu \mathrm{g} / \mathrm{kg}$ & 9 & 2 & 4.45 & \pm & 0.35 & $* *$ & 2.08 & \pm & 0.16 & $* *$ & 9.7 & \pm & 0.0 \\
\hline \multirow[t]{6}{*}{$\overline{\mathrm{EE}}$} & GD $14-17$ & Control & 30 & 4 & 6.73 & \pm & 0.60 & & 2.82 & \pm & 0.13 & & 13.6 & \pm & 2.1 \\
\hline & & $10 \mu \mathrm{g} / \mathrm{kg}$ & 26 & 4 & 6.16 & \pm & 0.52 & & 2.76 & \pm & 0.15 & & 11.1 & \pm & 1.2 \\
\hline & & $100 \mu \mathrm{g} / \mathrm{kg}$ & 37 & 5 & 6.58 & \pm & 0.19 & & 2.80 & \pm & 0.07 & & 13.0 & \pm & 1.0 \\
\hline & GD 18-21 & Control & 32 & 5 & 6.78 & \pm & 0.41 & & 2.81 & \pm & 0.15 & & 14.1 & \pm & 0.6 \\
\hline & & $10 \mu \mathrm{g} / \mathrm{kg}$ & 35 & 4 & 6.98 & \pm & 0.34 & & 3.00 & \pm & 0.16 & & 12.6 & \pm & 0.5 \\
\hline & & $100 \mu \mathrm{g} / \mathrm{kg}$ & 31 & 5 & 7.07 & \pm & 0.54 & & 2.89 & \pm & 0.22 & & 14.7 & \pm & 1.5 \\
\hline \multirow[t]{6}{*}{ TAM } & GD $14-17$ & Control & 30 & 4 & 6.73 & \pm & 0.60 & & 2.82 & \pm & 0.13 & & 13.6 & \pm & 2.1 \\
\hline & & $0.03 \mathrm{mg} / \mathrm{kg}$ & 25 & 4 & 6.50 & \pm & 0.88 & & 2.71 & \pm & 0.21 & & 13.8 & \pm & 2.7 \\
\hline & & $0.1 \mathrm{mg} / \mathrm{kg}$ & 9 & 2 & 6.52 & \pm & 0.19 & & 2.73 & \pm & 0.02 & & 13.7 & \pm & 0.9 \\
\hline & GD $18-21$ & Control & 32 & 5 & 6.78 & \pm & 0.41 & & 2.81 & \pm & 0.15 & & 14.1 & \pm & 0.6 \\
\hline & & $0.03 \mathrm{mg} / \mathrm{kg}$ & 16 & 3 & 6.84 & \pm & 0.06 & & 2.73 & \pm & 0.12 & & 15.8 & \pm & 1.7 \\
\hline & & $0.1 \mathrm{mg} / \mathrm{kg}$ & 25 & 4 & 6.57 & \pm & 0.46 & & 2.68 & \pm & 0.14 & & 15.0 & \pm & 2.6 \\
\hline
\end{tabular}

Value: Mean \pm S.D. calculated using the litter as the unit.

FLU: flutamide; DDE: $p, p^{\prime}$-dichlorodiphenyldichloroethylene; VZ: vinclozolin; DES: diethylstilbestrol; EE: ethynylestradiol; TAM: tamoxifen; GD: gestational day; PND: postnatal day.

Correction value of AGD: $\mathrm{AGD}(\mathrm{mm}) / \sqrt[3]{\text { body weight }(\mathrm{g})}$.

*: significantly different from control, $\mathrm{p}<0.05$; **: significantly different from control, $\mathrm{p}<0.01$. 
Table 4. Relative Organ Weights and Body Weights of PND 56 Male Rats Prenatally Exposed to Chemicals

\begin{tabular}{|c|c|c|c|c|c|c|c|c|c|c|c|}
\hline \multirow{2}{*}{$\begin{array}{l}\text { Chemical } \\
\text { Dosing period } \\
\text { FLU }\end{array}$} & \multirow[t]{2}{*}{ Group } & \multirow[t]{2}{*}{ Males } & \multirow[t]{2}{*}{ Litters } & $\begin{array}{c}\text { Body weight } \\
(\mathrm{g})\end{array}$ & \multicolumn{2}{|c|}{$\begin{array}{l}\text { Testes } \\
(\mathrm{mg} / \mathrm{g})\end{array}$} & \multicolumn{2}{|c|}{$\begin{array}{l}\text { Epididymides } \\
(\mathrm{mg} / \mathrm{g})\end{array}$} & \multicolumn{2}{|c|}{$\begin{array}{l}\text { Ventral prostate } \\
\quad(\mathrm{mg} / \mathrm{g})\end{array}$} & $\begin{array}{l}\text { Seminal Vesicles } \\
\quad(\mathrm{mg} / \mathrm{g})\end{array}$ \\
\hline & & & & & & & & & & & \\
\hline GD 14-17 & Control & 12 & 3 & $350.2 \pm 10.6$ & 8.674 & \pm 0.105 & 1.439 & \pm 0.054 & 0.791 & \pm 0.007 & $1.464 \pm 0.188$ \\
\hline & $1 \mathrm{mg} / \mathrm{kg}$ & 12 & 3 & $331.1 \pm 2.2$ & 8.433 & \pm 0.313 & 1.481 & \pm 0.026 & 0.829 & \pm 0.060 & $1.605 \pm 0.065$ \\
\hline & $10 \mathrm{mg} / \mathrm{kg}$ & 12 & 3 & $341.4 \pm 27.5$ & 7.982 & \pm 0.819 & 1.410 & \pm 0.091 & 0.818 & \pm 0.105 & $1.657 \pm 0.284$ \\
\hline GD 18-21 & Control & 12 & 3 & $333.0 \pm 15.5$ & 8.364 & \pm 0.084 & 1.499 & \pm 0.066 & 0.855 & \pm 0.023 & $1.651 \pm 0.227$ \\
\hline & $1 \mathrm{mg} / \mathrm{kg}$ & 12 & 3 & $350.0 \pm 21.9$ & 8.164 & \pm 0.115 & 1.456 & \pm 0.083 & 0.798 & \pm 0.143 & $1.694 \pm 0.164$ \\
\hline & $10 \mathrm{mg} / \mathrm{kg}$ & 7 & 3 & $346.6 \pm 29.3$ & 8.447 & \pm 0.438 & 1.460 & \pm 0.080 & 0.641 & \pm 0.115 & $1.642 \pm 0.278$ \\
\hline \multicolumn{12}{|l|}{$\overline{\mathrm{DDE}}$} \\
\hline \multirow[t]{4}{*}{ GD $14-17$} & Control & 12 & 3 & $350.2 \pm 10.6$ & 8.674 & \pm 0.105 & 1.439 & \pm 0.054 & 0.791 & \pm 0.007 & $1.464 \pm 0.188$ \\
\hline & $10 \mathrm{mg} / \mathrm{kg}$ & 12 & 3 & $359.1 \pm 38.6$ & 8.330 & \pm 0.125 & 1.447 & \pm 0.113 & 0.862 & 992 & $1.703 \pm 0.206$ \\
\hline & $30 \mathrm{mg} / \mathrm{kg}$ & 16 & 4 & $368.9 \pm 38.3$ & 7.763 & $\pm 0.492 * *$ & 1.431 & \pm 0.062 & 0.946 & \pm 0.082 & $1.672 \pm 0.201$ \\
\hline & $100 \mathrm{mg} / \mathrm{kg}$ & 16 & 4 & $340.4 \pm 23.4$ & 8.785 & \pm 0.229 & 1.465 & \pm 0.097 & 0.743 & \pm 0.102 & $1.601 \pm 0.280$ \\
\hline \multirow[t]{4}{*}{ GD 18-21 } & Control & 14 & 4 & $338.5 \pm 15.6$ & 8.291 & \pm 0.374 & 1.444 & \pm 0.095 & 0.882 & \pm 0.062 & $1.817 \pm 0.032$ \\
\hline & $10 \mathrm{mg} / \mathrm{kg}$ & 16 & 4 & $342.9 \pm 15.6$ & 8.308 & \pm 0.186 & 1.490 & \pm 0.058 & 0.758 & \pm 0.052 & $1.794 \pm 0.270$ \\
\hline & $30 \mathrm{mg} / \mathrm{kg}$ & 14 & 4 & $352.7 \pm 21.6$ & 7.927 & \pm 0.285 & 1.443 & \pm 0.075 & 0.903 & 120 & $1.785 \pm 0.142$ \\
\hline & $100 \mathrm{mg} / \mathrm{kg}$ & 4 & 1 & 339.9 & 9.027 & & 1.582 & & 1.003 & & 1.816 \\
\hline \multicolumn{12}{|l|}{$\overline{V Z}$} \\
\hline \multirow[t]{4}{*}{ GD $14-17$} & $\mathrm{Co}$ & 20 & 5 & $355.2 \pm 19.5$ & 7.177 & \pm 0.212 & 1.463 & \pm 0.073 & 0.982 & 089 & $1.729 \pm 0.262$ \\
\hline & $10 \mathrm{mg} / \mathrm{kg}$ & 19 & 5 & $352.9 \pm 6.1$ & 7.486 & \pm 0 & 1.514 & \pm 0.080 & 0.931 & & $1.752 \pm 0.068$ \\
\hline & $30 \mathrm{mg} / \mathrm{kg}$ & 19 & 5 & $344.6 \pm 18.9$ & 7.809 & \pm 0 & 1.489 & \pm 0.139 & 0.992 & 124 & $1.840 \pm 0.308$ \\
\hline & $100 \mathrm{mg} / \mathrm{kg}$ & 20 & 1 & $369.2 \pm 6.4$ & 7.404 & \pm 0.608 & 1.489 & \pm 0.062 & 0.755 & $\pm 0.093 * *$ & $1.675 \pm 0.213$ \\
\hline \multirow[t]{4}{*}{ GD 18-21 } & Control & 20 & 5 & $363.8 \pm 49.2$ & 7.766 & \pm 0.955 & 1.523 & \pm 0.169 & 0.970 & \pm 0.131 & $1.869 \pm 0.210$ \\
\hline & $10 \mathrm{mg} / \mathrm{kg}$ & 16 & 4 & $358.5 \pm 32.8$ & 7.768 & \pm 0.493 & 1.596 & \pm 0.166 & 1.007 & \pm 0.178 & $1.996 \pm 0.327$ \\
\hline & $30 \mathrm{mg} / \mathrm{kg}$ & 16 & 4 & $357.4 \pm 16.1$ & 7.525 & \pm 0.405 & 1.597 & \pm 0.084 & 0.934 & \pm 0.095 & $1.830 \pm 0.339$ \\
\hline & $100 \mathrm{mg} / \mathrm{kg}$ & 15 & 4 & $357.2 \pm 19.7$ & 7.609 & \pm 0.309 & 1.517 & \pm 0.066 & 0.963 & & $1.771 \pm 0.119$ \\
\hline \multicolumn{12}{|l|}{$\overline{\mathrm{DES}}$} \\
\hline \multirow[t]{6}{*}{ GD $14-17$} & Control & 19 & 5 & $347.0 \pm 23.9$ & 7.980 & \pm 1.152 & 1.454 & \pm 0.106 & 0.983 & 132 & $1.922 \pm 0.086$ \\
\hline & $0.1 \mu \mathrm{g} / \mathrm{kg}$ & 12 & 3 & $353.6 \pm 11.7$ & 7.802 & \pm 0.485 & 1.581 & \pm 0.043 & 1.091 & & $1.902 \pm 0.239$ \\
\hline & $1 \mu \mathrm{g} / \mathrm{kg}$ & 20 & 5 & $358.3 \pm 22.7$ & 7.639 & \pm 0.619 & 1.527 & \pm 0.105 & 1.004 & 081 & $1.850 \pm 0.092$ \\
\hline & $10 \mu \mathrm{g} / \mathrm{kg}$ & 16 & 4 & $363.0 \pm 23.6$ & 7.966 & \pm 0.554 & 1.626 & \pm 0.088 & 1.037 & \pm 0.102 & $2.120 \pm 0.187$ \\
\hline & $100 \mu \mathrm{g} / \mathrm{kg}$ & 16 & 4 & $373.5 \pm 13.7$ & 7.337 & \pm 0.523 & 1.532 & \pm 0.084 & 0.913 & & $1.888 \pm 0.243$ \\
\hline & $300 \mu \mathrm{g} / \mathrm{kg}$ & 12 & 4 & $350.4 \pm 20.1$ & 7.888 & \pm 0.320 & 1.592 & \pm 0.062 & 0.718 & $58 * *$ & $1.885 \pm 0.251$ \\
\hline GD 18-21 & Control & 16 & 4 & $343.8 \pm 11.2$ & 8.006 & \pm 0.610 & 1.609 & \pm 0.102 & 1.115 & \pm 0.040 & $1.872 \pm 0.144$ \\
\hline & $0.1 \mu \mathrm{g} / \mathrm{kg}$ & 20 & 5 & $353.3 \pm 20.7$ & 8.369 & \pm 1.418 & 1.491 & \pm 0.072 & 1.100 & \pm 0 & $1.853 \pm 0.263$ \\
\hline & $1 \mu \mathrm{g} / \mathrm{kg}$ & 16 & 4 & $370.1 \pm 25.7$ & 7.110 & \pm 0.642 & 1.517 & \pm 0.161 & 1.033 & \pm 0.094 & $1.866 \pm 0.238$ \\
\hline & $10 \mu \mathrm{g} / \mathrm{kg}$ & 20 & 5 & $354.8 \pm 15.0$ & 7.959 & \pm 0.389 & 1.633 & \pm 0.116 & 1.050 & & $1.974 \pm 0.290$ \\
\hline & $\mathrm{g} / \mathrm{kg}$ & 20 & 5 & $354.9 \pm 10.2$ & 7 & \pm 0 & 1.577 & \pm 0.079 & 0.753 & $1 * *$ & $1.743 \pm 0.136$ \\
\hline & $\mathrm{g} / \mathrm{kg}$ & 7 & 2 & $316.5 \pm 2.8$ & 8.228 & \pm 0 . & 1.664 & \pm 0.025 & 0.668 & \pm & $1.991 \pm 0.364$ \\
\hline$\overline{\mathrm{EE}}$ & & & & & & & & & & & \\
\hline GD $14-17$ & $\mathrm{Co}$ & 16 & 4 & $364.1 \pm 19.7$ & 7.912 & \pm 0.032 & 1.583 & \pm 0.122 & 0.954 & \pm 0.095 & $1.918 \pm 0.116$ \\
\hline & $10 \mu \mathrm{g} / \mathrm{kg}$ & 16 & 4 & $333.8 \pm 21.5$ & 7.693 & \pm 0.280 & 1.477 & \pm 0.076 & 0.967 & 145 & $1.877 \pm 0.185$ \\
\hline & $100 \mu \mathrm{g} / \mathrm{kg}$ & 20 & 5 & $354.9 \pm 17.0$ & 7.745 & \pm 0.189 & 1.538 & \pm 0.086 & 0.980 & \pm 0.165 & $1.993 \pm 0.197$ \\
\hline GD 18-21 & Control & 19 & 5 & $358.5 \pm 14.1$ & 7.677 & \pm 0.424 & 1.477 & \pm 0.112 & 0.949 & \pm 0.092 & $1.967 \pm 0.185$ \\
\hline & $10 \mu \mathrm{g} / \mathrm{kg}$ & 15 & 4 & $372.0 \pm 16.2$ & 7.752 & \pm 0.454 & 1.539 & \pm 0.073 & 1.036 & \pm 0.124 & $2.106 \pm 0.062$ \\
\hline & $100 \mu \mathrm{g} / \mathrm{kg}$ & 16 & 4 & $364.8 \pm 6.2$ & 7.791 & \pm 0.475 & 1.618 & \pm 0.062 & 0.923 & \pm 0.089 & $2.070 \pm 0.173$ \\
\hline$\overline{\text { TAM }}$ & & & & & & & & & & & \\
\hline GD $14-17$ & Cont & 16 & 4 & $364.1 \pm 19.7$ & 7.912 & \pm 0.032 & 1.583 & \pm 0.122 & 0.954 & 995 & $1.918 \pm 0.116$ \\
\hline & $0.03 \mathrm{mg} / \mathrm{kg}$ & 15 & 4 & $356.8 \pm 26.7$ & 7.808 & \pm 0.311 & 1.491 & \pm 0.116 & 0.996 & \pm & $1.948 \pm 0.285$ \\
\hline & $0.1 \mathrm{mg} / \mathrm{kg}$ & 7 & 2 & $360.7 \pm 13.4$ & 7.320 & \pm 0.038 & 1.458 & \pm 0.117 & 0.903 & \pm 0.014 & $1.659 \pm 0.076$ \\
\hline GD 18-21 & Control & 19 & 5 & $358.5 \pm 14.1$ & 7.677 & \pm 0.424 & 1.477 & \pm 0.112 & 0.949 & \pm 0.092 & $1.967 \pm 0.185$ \\
\hline & $0.03 \mathrm{mg} / \mathrm{kg}$ & 12 & 3 & $359.8 \pm 44.0$ & 7.142 & \pm 0.660 & 1.469 & \pm 0.030 & 0.932 & \pm 0.174 & $1.869 \pm 0.277$ \\
\hline & $0.1 \mathrm{mg} / \mathrm{kg}$ & 11 & 3 & $376.4 \pm 49.8$ & 7.475 & \pm 0.202 & 1.513 & \pm 0.020 & 0.949 & \pm 0.036 & $1.731 \pm 0.093$ \\
\hline
\end{tabular}

Value: Mean \pm S.D. calculated using the litter as the unit.

FLU: flutamide; DDE: $p, p^{\prime}$-dichlorodiphenyldichloroethylene; VZ: vinclozolin; DES: diethylstilbestrol; EE: ethynylestradiol; TAM: tamoxifen; GD: gestational day; PND: postnatal day.

**: significantly different from control, $\mathrm{p}<0.01$. 


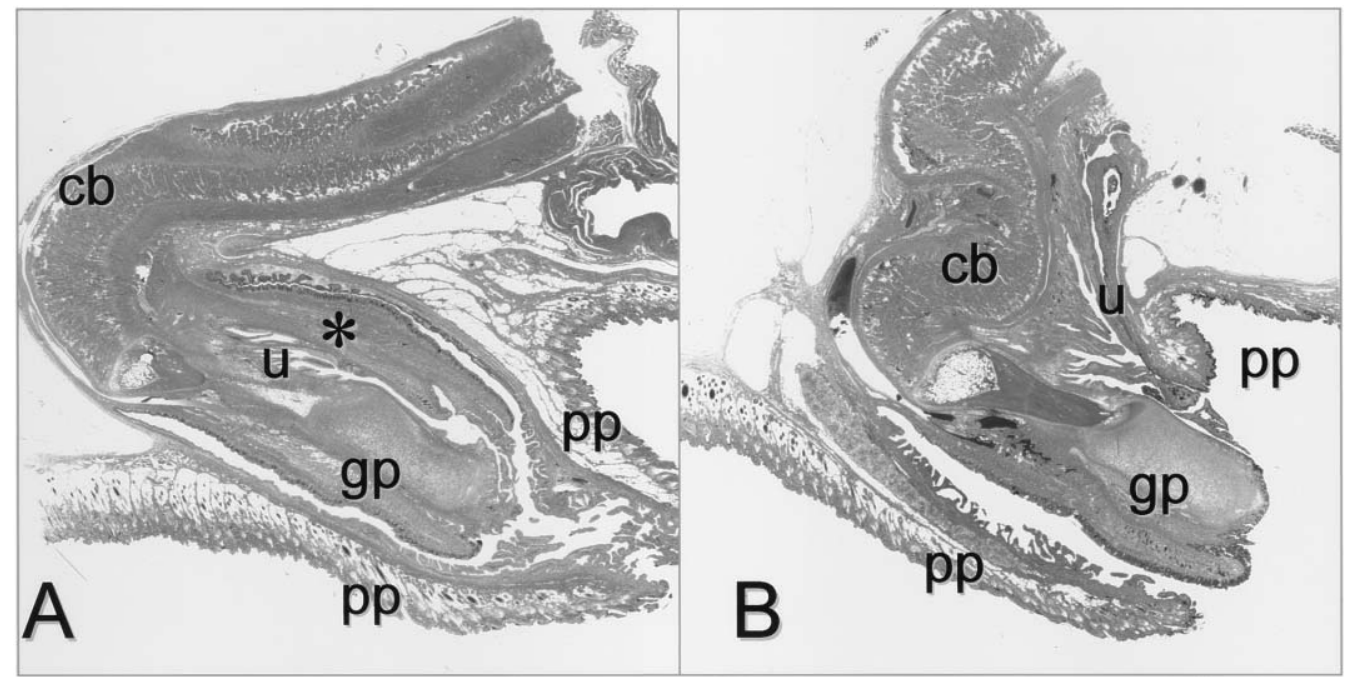

Fig. 3. Sagittal sections of the prepuce and penis of male rats sacrificed on PND 56.

A: Control male rat. The prepuce (pp) is separated from the glans penis (gp). The urethra (u) is located in the center of the glans penis. *: ventral half of the glans penis. cb: cavernous body. H\&E staining, magnification: $\times 6$. B: Male rat from a dam exposed to $100 \mathrm{mg} / \mathrm{kg}$ vinclozolin on GD 14-17. The prepuce (pp) is separated from the glans penis (gp) at the dorsal part, and the urethra $(\mathrm{u})$ is located between the glans penis and subcutis. The prepuce is hypoplastic and the glans penis is not completely covered by the prepuce. The cavernous body $(\mathrm{cb})$ of the penis is tortuous and bent. H\&E staining, magnification: $\times 6$.

difference in AGD. Body weights at the time of AGD measurement did not show any significant difference.

\section{5) Organ weight}

Relative organ weights of males prenatally exposed to chemicals are shown in Table 4. Changes in absolute organ weights were similar to changes in relative organ weights. Relative weights of the ventral prostate were decreased significantly in males from dams exposed to $100 \mathrm{mg} / \mathrm{kg}$ of VZ on GD 14-17, $300 \mu \mathrm{g} / \mathrm{kg}$ of DES on GD 14-17, and 100 and $300 \mu \mathrm{g} / \mathrm{kg}$ of DES on GD 18-21. Although there was a significant decrease in relative organ weight of the testes in the DDE group, a dose dependent relationship was not detected.

\section{6) Pathological examination}

Histopathological examination of representative males from dams exposed to $10 \mathrm{mg} / \mathrm{kg}$ of FLU or $100 \mathrm{mg} / \mathrm{kg}$ of VZ on GD 14-17 revealed a defect in the ventral half of the glans penis (cleft phallus). The urethra was not located in the center of the glans penis, but instead was observed at the ventral surface of the penis (Fig. 3B). The dorsal surface of the glans penis and prepuce of PND 56 males were covered with keratinized stratified squamous epithelium, and the prepuce was separated from the glans penis. The ventral part of the glans penis and ventral epithelium were not formed between the urethra and subcutis, the ventral surface of the glans penis was not covered with squamous epithelium, and preputial separation did not progress at the ventral aspect. The external urethral orifice opened at the ventral surface of the penis (hypospadias). These males with cleft phallus showed a tortuous cavernous body of the penis (Fig. 3B). On PND 6, hypoplasia of the ventral half of the glans penis and tortuous cavernous body were observed in male pups from dams exposed to $100 \mathrm{mg} / \mathrm{kg}$ of $\mathrm{VZ}$ on GD 14-17 (Fig. 4B).

Ectopic testes were induced by FLU and VZ, and showed severe atrophy of the seminiferous tubule in PND 56 males. Severe prostatitis and seminal vesiculitis were observed in males from dams exposed to $10 \mathrm{mg} / \mathrm{kg}$ of FLU on GD 18-21. Neutrophils, lymphocytes and macrophages infiltrated the prostate, seminal vesicles, and surrounding tissues of five animals which died (PND 44, 45, 52, 55 and 56) and two sacrificed (PND 56) males. In some cases, hemorrhage was observed in the muscular layer of the urinary bladder. The males with severe prostatitis and vesiculitis did not show hypoplasia in the ventral half of the glans penis.

\section{Postnatal exposure \\ 1) Preputial separation}

The day of preputial separation and the body weights on the day of preputial separation were statistically analyzed using both the litter as the unit and the individual data. There were no significant changes on the day of preputial separation of males exposed to FLU, DDE or VZ on PND 15 (Table 5). On the other hand, the day of preputial separation was significantly delayed in groups exposed to 10 and $30 \mathrm{mg} / \mathrm{kg}$ of FLU and $30 \mathrm{mg} / \mathrm{kg}$ of VZ on PND 35-39. Statistical analyses using the individual data revealed additional significances in the delay of preputial separation in the groups exposed $300 \mathrm{mg} / \mathrm{kg}$ of DDE on PND 17-21 and $100 \mathrm{mg} / \mathrm{kg}$ on PND 35-39, and $100 \mathrm{mg} / \mathrm{kg}$ of VZ on PND 35-39. In the DDE group, 10 of 12 males exposed to 300 $\mathrm{mg} / \mathrm{kg}$ of DDE on PND 35-39 died before maturation. 


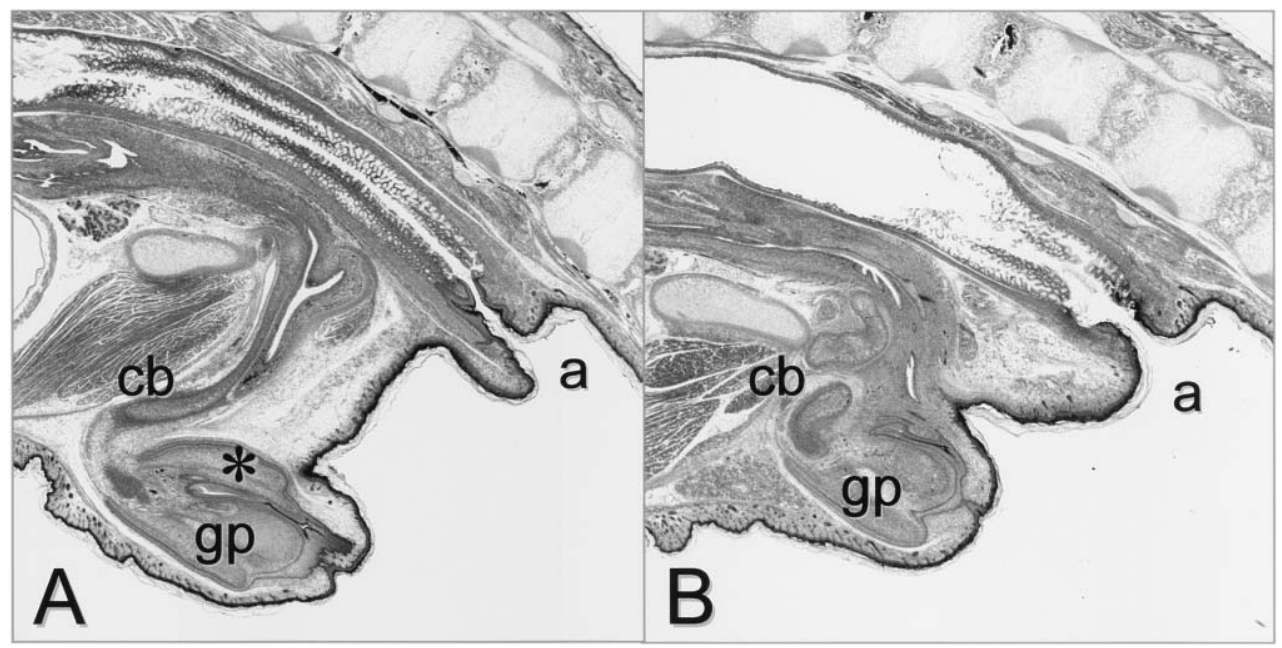

Fig. 4. Sagittal sections of the genital tubercle of males sacrificed on PND 6.

A: Control male rat. Glans penis (gp) is bordered with dorsal and ventral epithelium. The urethra is located in the center of the glans penis. a: anus. *: ventral half of the glans penis. H\&E staining, magnification: $\times 8$. B: A male rat from a dam exposed to $100 \mathrm{mg} / \mathrm{kg}$ vinclozolin on GD 14-17. The dorsal part of the glans penis is bordered with epithelium. The urethra is observed at the ventral surface of the glans penis (gp) and the cavernous body (cb) shows tortuous structure. a: anus. H\&E staining, magnification: $\times 8$.

Preputial separation in two surviving males (PND 48 and 50) was delayed compared to controls. A significant delay was not detected in males exposed to FLU or VZ on PND 17-21.

DES induced a significant delay in the $300 \mu \mathrm{g} / \mathrm{kg}$ group exposed on PND 1-5 or PND 35-39, while 2 of 16 males in the $100 \mu \mathrm{g} / \mathrm{kg}$ group and 3 of 16 males in the $300 \mu \mathrm{g} / \mathrm{kg}$ group exposed on PND 1-5 showed incomplete preputial separation on PND 56. In statistical analyses using the individual data, significant delays of preputial separation were also observed in the group exposed to $100 \mu \mathrm{g} / \mathrm{kg}$ of DES on PND 1-5 or 35-39. There were no significant delays in males exposed on PND 17-21.

In EE treatment, preputial separation was significantly delayed in males of the $100 \mu \mathrm{g} / \mathrm{kg}$ group exposed on PND 15 , and 1 of 12 males in the $10 \mu \mathrm{g} / \mathrm{kg}$ group and 10 of 12 males in the $100 \mu \mathrm{g} / \mathrm{kg}$ group had incomplete separation on PND 56. Administration of $10 \mu \mathrm{g} / \mathrm{kg}$ of EE on PND 6-10 or PND 11-15 was added to the experimental protocol to define the sensitive period, because PND 1-5 exposure induced a marked effect on preputial separation. Although a delay in preputial separation was observed after PND 6-10 exposure, there was no significant difference between the PND 11-15 exposed group and controls. The $3 \mathrm{mg} / \mathrm{kg}$ TAM treated groups exposed on PND 1-5 also showed a significant delay in preputial separation, and 5 of 16 males in the $1 \mathrm{mg} / \mathrm{kg}$ group and 14 of 15 males in the $3 \mathrm{mg} / \mathrm{kg}$ group had incomplete separation on PND 56. Slight delay in preputial separation was also observed in the $0.3 \mathrm{mg} / \mathrm{kg}$ TAM group, and 2 of 16 males had incomplete separation on PND 56. In the statistical analyses using the individual data, significant delays of preputial separation were also observed in the groups exposed to $10 \mu \mathrm{g} / \mathrm{kg}$ of EE and $1 \mathrm{mg} / \mathrm{kg}$ of TAM on
PND 1-5. No influence of EE and TAM treatments on PND 17-21 or PND 35-39 was detected.

Body weights on the day of preputial separation showed a higher value in the groups with a delay of separation.

\section{2) Organ weight}

Relative organ weights of males postnatally exposed to the chemicals are presented in Table 6 using the litter as the unit and in Table 7 using the individual data. Absolute organ weights showed similar changes to those of relative organ weights. Relative weights of the ventral prostate were decreased significantly in males exposed to $30 \mathrm{mg} / \mathrm{kg}$ of FLU on PND 35-39. No significant changes were observed in males exposed to FLU on PND 1-5 or 17-21, and DDE and $\mathrm{VZ}$ in any period.

DES caused a significant reduction in the relative weight of the ventral prostate in the 100 and $300 \mu \mathrm{g} / \mathrm{kg}$ groups exposed on PND 1-5. No significant changes were observed in males exposed to DES on PND 17-21 or PND 35-39. EE caused reductions in the testes, ventral prostate and seminal vesicles of males in the $100 \mu \mathrm{g} / \mathrm{kg}$ group and seminal vesicles of males in the $10 \mu \mathrm{g} / \mathrm{kg}$ group exposed to EE on PND 1-5. No significant changes in relative weight were observed in males exposed on PND 17-21 or PND 3539. TAM treatment on PND 1-5 led to reductions in body and reproductive organ weights in the $3 \mathrm{mg} / \mathrm{kg}$ group and the ventral prostate weight in the $1 \mathrm{mg} / \mathrm{kg}$ group. No weight reductions were observed in the reproductive organs of males exposed to TAM on PND 17-21 or PND 35-39. Although the statistical analyses using the individual data showed significant results in the lower dose group and the other organs, there were no significances in the groups exposed to VZ. 
Table 5. Preputial Separation and Body Weights of Male Rats Postnatally Exposed to Chemicals

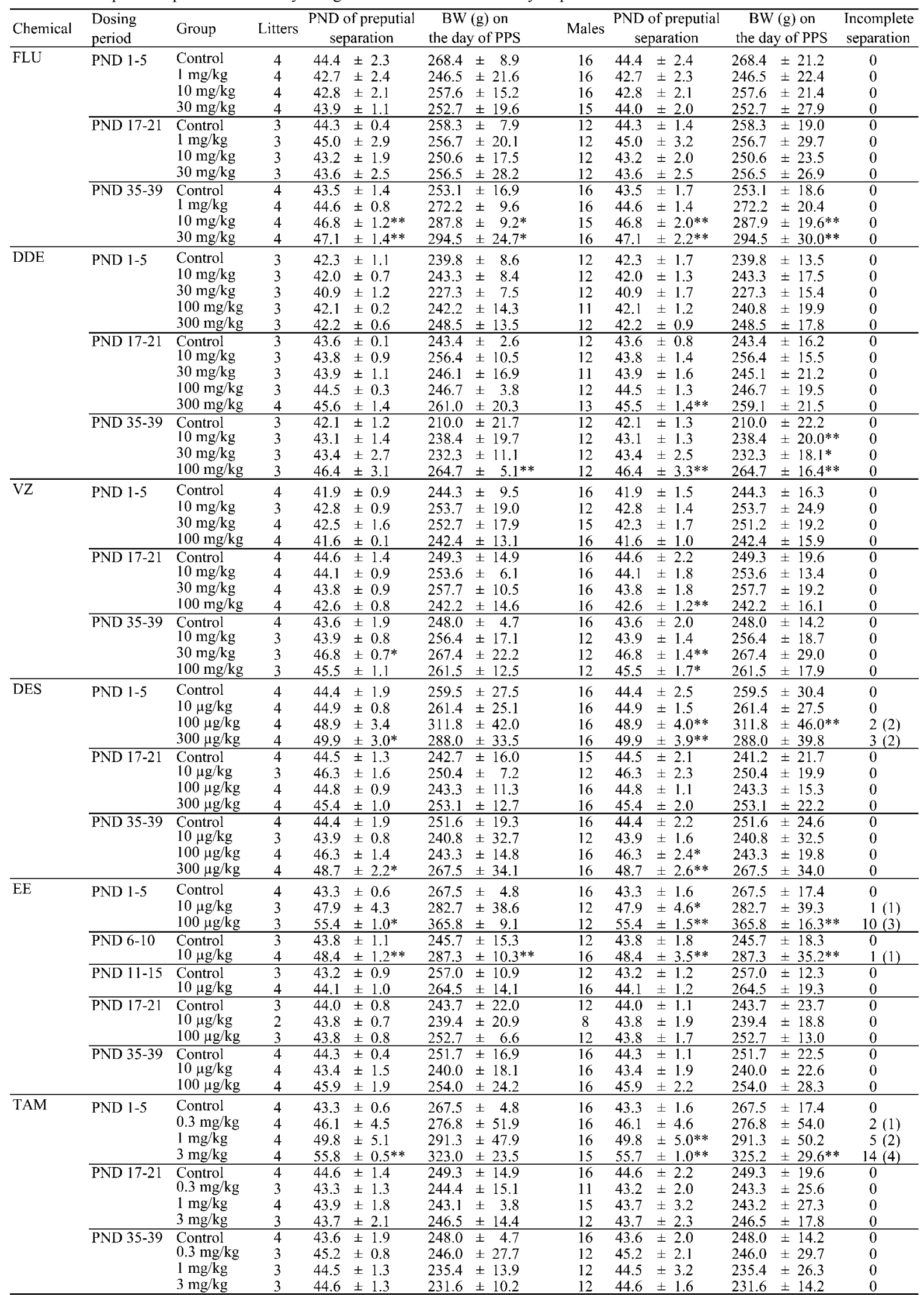

Value: Mean \pm S.D. using the litter as the unit or using individual data.

PND: postnatal day; PPS: preputial separation; FLU: flutamide; DDE: $p, p^{\prime}$-dichlorodiphenyldichloroethylene; VZ: vinclozolin; DES: diethylstilbestrol; EE: ethynylestradiol; TAM: tamoxifen; BW: body weight.

Incomplete separation: number of animals (litters) with incomplete separation on PND 56.

*: significantly different from control, $\mathrm{p}<0.05 ; * *$ : significantly different from control, $\mathrm{p}<0.01$. 
Table 6. Relative Organ Weights and Body Weights of PND 56 Male Rats Postnatally Exposed to Chemicals (Using the Litter as the Unit)

\begin{tabular}{|c|c|c|c|c|c|c|c|c|c|c|c|}
\hline \multicolumn{2}{|c|}{$\begin{array}{l}\text { Chemical } \\
\text { Dosing period }\end{array}$} & \multirow{2}{*}{$\begin{array}{l}\text { Group } \\
\text { Control }\end{array}$} & \multirow{2}{*}{$\begin{array}{c}\text { Litters } \\
4\end{array}$} & $\begin{array}{l}\text { Body weight } \\
\text { (g) }\end{array}$ & \multicolumn{2}{|c|}{$\begin{array}{l}\text { Testes } \\
(\mathrm{mg} / \mathrm{g})\end{array}$} & \multicolumn{2}{|c|}{$\begin{array}{l}\text { Epididymides } \\
(\mathrm{mg} / \mathrm{g})\end{array}$} & $\begin{array}{l}\text { Ventral prostate } \\
(\mathrm{mg} / \mathrm{g})\end{array}$ & Semin & $\begin{array}{l}1 \text { Vesicles } \\
\mathrm{g} / \mathrm{g})\end{array}$ \\
\hline FLU & PND 1-5 & & & $384.1 \pm 19.6$ & 7.281 & \pm 0.714 & 1.400 & \pm 0.079 & $1.025 \pm 0.137$ & 2.008 & \pm 0.289 \\
\hline & & $1 \mathrm{mg} / \mathrm{kg}$ & 4 & $378.1 \pm 15.6$ & 7.536 & \pm 0.457 & 1.511 & \pm 0.121 & $0.986 \pm 0.175$ & 1.895 & \\
\hline & & $10 \mathrm{mg} / \mathrm{kg}$ & 4 & $389.8 \pm 28.3$ & 7.441 & \pm 0.713 & 1.443 & \pm 0.074 & $0.998 \pm 0.112$ & 1.774 & \pm 0.216 \\
\hline & & $30 \mathrm{mg} / \mathrm{kg}$ & 4 & $371.4 \pm 17.0$ & 7.594 & \pm 0.583 & 1.438 & \pm 0.087 & $1.050 \pm 0.099$ & 1.806 & \pm 0.244 \\
\hline & $\overline{\text { PND } 17-21}$ & Control & 3 & $376.2 \pm 5.9$ & 7.446 & \pm 0.481 & 1.438 & \pm 0.082 & $0.989 \pm 0.051$ & 1.758 & \pm 0.220 \\
\hline & & $1 \mathrm{mg} / \mathrm{kg}$ & 3 & $358.8 \pm 11.3$ & 7.389 & \pm 0.075 & 1.489 & \pm 0.214 & $1.007 \pm 0.093$ & 1.835 & 266 \\
\hline & & $10 \mathrm{mg} / \mathrm{kg}$ & 3 & $372.1 \pm 14.1$ & 7.508 & \pm 0.469 & 1.451 & \pm 0.062 & $0.951 \pm 0.188$ & 1.918 & \pm 0.064 \\
\hline & & $30 \mathrm{mg} / \mathrm{kg}$ & 3 & $376.4 \pm 16.4$ & 7.707 & \pm 0.172 & 1.484 & \pm 0.040 & $1.043 \pm 0.041$ & 1.923 & 0.349 \\
\hline & 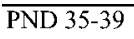 & Control & 4 & $377.3 \pm 30.6$ & 7.654 & \pm 0.302 & 1.520 & \pm 0.039 & $1.068 \pm 0.076$ & 2.065 & \pm 0.132 \\
\hline & & $1 \mathrm{mg} / \mathrm{kg}$ & 4 & $382.8+15.8$ & 8.108 & \pm 0.773 & 1.603 & \pm 0.153 & $1.097 \pm 0.112$ & 2.108 & \pm 0.099 \\
\hline & & $10 \mathrm{mg} / \mathrm{kg}$ & 4 & $378.5 \pm 19.9$ & 7.742 & \pm 0.725 & 1.407 & \pm 0.118 & $1.051 \pm 0.121$ & 1.774 & \pm 0.235 \\
\hline & & $30 \mathrm{mg} / \mathrm{kg}$ & 4 & $381.1 \pm 15.5$ & 7.951 & \pm 0.378 & 1.398 & \pm 0.093 & $0.866 \pm 0.106^{*}$ & 1.788 & \pm 0.131 \\
\hline$\overline{\mathrm{DDE}}$ & PND & Control & 3 & $362.5 \pm 6.2$ & 7.760 & \pm 0 . & 1.447 & \pm 0 & $0.809 \pm 0.084$ & 1.942 & 0.217 \\
\hline & & $10 \mathrm{mg} / \mathrm{kg}$ & 3 & $382.2 \pm 10.3$ & 7.122 & \pm 0 & 1.395 & 7 & $0.852 \pm 0$ & 1.874 & \\
\hline & & $30 \mathrm{mg} / \mathrm{kg}$ & 3 & $367.2 \pm 27.2$ & 7.670 & \pm 0 . & 1.443 & +0 & $0.928 \pm 0$ & 1.893 & 259 \\
\hline & & $100 \mathrm{mg} / \mathrm{kg}$ & 3 & $369.8 \pm 19.6$ & 7.458 & \pm 0 & 1.421 & +0 & $0.826 \pm 0$ & 1.792 & 049 \\
\hline & & $300 \mathrm{mg} / \mathrm{kg}$ & 3 & $374.0 \pm 13.3$ & 8.024 & \pm 0 . & 1.398 & \pm 0 & $0.749 \pm 0$ & 1.783 & 185 \\
\hline & $\overline{P N D} 17-21$ & Control & 3 & $358.6 \pm 11.8$ & 8.522 & \pm 0.9 & 1.539 & \pm 0.052 & $0.815 \pm 0.136$ & 1.724 & 319 \\
\hline & & $10 \mathrm{~m}$ & 3 & $377.0 \pm 7.0$ & 7.804 & \pm 0 . & 1.465 & & $393 \pm 0$ & 1.820 & 284 \\
\hline & & $30 \mathrm{mg} / \mathrm{kg}$ & 3 & $359.7 \pm 12.9$ & 7.864 & \pm 0 . & 1.497 & +0 & $0.864 \pm 0$ & 1.674 & 171 \\
\hline & & $100 \mathrm{mg} / \mathrm{kg}$ & 3 & $362.8 \pm 2.4$ & 8.263 & \pm 0 . & 1.502 & +0 & $0.840 \pm 0$ & 1.751 & 095 \\
\hline & & $300 \mathrm{mg} / \mathrm{kg}$ & 4 & $361.9 \pm 33.6$ & 8.344 & \pm 0 . & 1.423 & +0 & $848 \pm 0$ & 1.550 & 14 \\
\hline & PND 35-39 & Control & 3 & $333.5 \pm 26.1$ & 7.844 & \pm 0 & 1.520 & - & $0.925 \pm 0$ & 1.834 & 18 \\
\hline & & /kg & 3 & $356.0 \pm 22.3$ & 7.362 & \pm 0 & 1.323 & & $375 \pm 0$ & 1.831 & 10 \\
\hline & & $30 \mathrm{mg} / \mathrm{kg}$ & 3 & $347.2 \pm 40.7$ & 7.500 & \pm 0 . & 1.376 & & $0.908 \pm 0$ & 1.807 & 172 \\
\hline & & $100 \mathrm{mg} / \mathrm{kg}$ & 3 & $353.5 \pm 23.0$ & 7.823 & \pm 0.247 & 1.409 & \pm 0.108 & $0.847 \pm 0.065$ & 1.523 & \pm 0.249 \\
\hline $\mathrm{VZ}$ & PND 1-5 & Control & 4 & $367.5 \pm 20.6$ & 7.990 & \pm 0 . & 1.601 & \pm 0.142 & $1.049 \pm 0.151$ & 2.151 & 180 \\
\hline & & $10 \mathrm{mg} / \mathrm{kg}$ & 3 & $382.0 \pm 32.7$ & 7.392 & \pm 0 . & 1.477 & \pm 0 & $1.006 \pm 0$ & 2.059 & 083 \\
\hline & & $30 \mathrm{mg} / \mathrm{kg}$ & 4 & $380.1 \pm 9.5$ & 7.986 & \pm 0 & 1.500 & \pm 0 . & $0.910 \pm 0.118$ & 2.049 & \pm 0.093 \\
\hline & & $100 \mathrm{mg} / \mathrm{kg}$ & 4 & $377.9 \pm 17.6$ & 7.373 & \pm 0.152 & 1.582 & \pm 0.109 & $1.030 \pm 0.094$ & 2.116 & \pm 0.082 \\
\hline & $\overline{\text { PND } 17-21}$ & Control & 4 & $357.5 \pm 26.3$ & 7.791 & \pm 0.654 & 1.432 & \pm 0.058 & $0.967 \pm 0.094$ & 1.903 & \pm 0.300 \\
\hline & & $\mathrm{g} / \mathrm{kg}$ & 4 & $366.8 \pm 9.9$ & 7.981 & \pm 0 & 1.562 & \pm 0. & $0.987 \pm 0.018$ & 2.041 & 39 \\
\hline & & $30 \mathrm{mg} / \mathrm{kg}$ & 4 & $378.3 \pm 17.4$ & 7.629 & \pm 0.610 & 1.550 & \pm 0.147 & $0.891 \pm 0.105$ & 1.931 & \pm 0.237 \\
\hline & & $100 \mathrm{mg} / \mathrm{kg}$ & 4 & $373.2 \pm 25.5$ & 8.066 & \pm 0.507 & 1.532 & $\begin{array}{l}0.137 \\
\end{array}$ & $1.018 \pm 0.103$ & 2.105 & 135 \\
\hline & 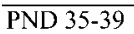 & Control & 4 & $369.8 \pm 20.8$ & 7.711 & \pm 0.696 & 1.502 & \pm 0.053 & $0.895 \pm 0.041$ & 1.752 & 0.115 \\
\hline & & $\mathrm{g} / \mathrm{kg}$ & 3 & $373.3 \pm 39.0$ & 7.420 & \pm 0 & 1.572 & \pm 0 . & $0.910 \pm 0.0$ & 1.911 & 81 \\
\hline & & $30 \mathrm{mg} / \mathrm{kg}$ & 3 & $361.7 \pm 32.0$ & 8.125 & \pm 0.2 & 1.546 & \pm 0.0 & $0.982 \pm 0.042$ & 1.885 & \pm 0.053 \\
\hline & & $100 \mathrm{mg} / \mathrm{kg}$ & 3 & $363.1 \pm 5.5$ & 7.719 & \pm 0.280 & 1.508 & $\begin{array}{r}0.088 \\
\end{array}$ & $0.941 \pm 0.058$ & 1.907 & \pm 0.083 \\
\hline DES & PND 1-5 & Control & 4 & $367.5 \pm 25.0$ & 8.089 & \pm 0 . & 1.560 & \pm 0.151 & $1.064 \pm 0.097$ & 1.948 & .141 \\
\hline & & /kg & 4 & $365.7 \pm 24.8$ & 7.296 & \pm 0.588 & 391 & \pm 0.100 & $0.917 \pm 0$. & 1.870 & 64 \\
\hline & & $100 \mu \mathrm{g} / \mathrm{kg}$ & 4 & $383.6 \pm 13.7$ & 8.277 & \pm 1.331 & 1.431 & \pm 0.121 & $0.769 \pm 0.088^{* *}$ & 1.668 & \pm 0.537 \\
\hline & & $300 \mu \mathrm{g} / \mathrm{kg}$ & 4 & $343.6 \pm 19.7$ & 7.840 & \pm 1.413 & 1.585 & \pm 0.206 & $0.829 \pm 0.076^{*}$ & 1.323 & \pm 0.086 \\
\hline & $\overline{\text { PND } 17-21}$ & Control & 4 & $343.3 \pm 32.2$ & 7.753 & \pm 0.315 & 1.554 & \pm 0.155 & $0.925 \pm 0.046$ & 1.952 & \pm 0.302 \\
\hline & & $10 \mu \mathrm{g} / \mathrm{kg}$ & 3 & $337.2 \pm 20.4$ & 7.906 & \pm 0.530 & 1.432 & \pm 0.075 & $0.922 \pm 0.083$ & 1.781 & \pm 0.050 \\
\hline & & $100 \mu \mathrm{g} / \mathrm{kg}$ & 4 & $348.9 \pm 19.6$ & 6.940 & \pm 0.696 & 1.455 & \pm 0.105 & $0.900 \pm 0.095$ & 1.939 & \pm 0.281 \\
\hline & & $300 \mu \mathrm{g} / \mathrm{kg}$ & 4 & $352.8 \pm 13.8$ & 7.309 & \pm 0.751 & 1.417 & \pm 0.093 & $0.933 \pm 0.099$ & 1.935 & \pm 0.317 \\
\hline & PND 35-39 & Control & 4 & $363.7 \pm 38.2$ & 7.750 & \pm 0.452 & 1.542 & \pm 0.103 & $1.052 \pm 0.045$ & 2.303 & \pm 0.295 \\
\hline & & $10 \mu \mathrm{g} / \mathrm{kg}$ & 3 & $344.9 \pm 40.5$ & 7.472 & \pm 0.696 & 1.593 & \pm 0.146 & $1.035 \pm 0.113$ & 1.963 & \pm 0.221 \\
\hline & & $100 \mu \mathrm{g} / \mathrm{kg}$ & 4 & $330.4 \pm 23.8$ & 7.920 & \pm 0.561 & 1.556 & \pm 0.024 & $0.987 \pm 0.160$ & 1.910 & \pm 0.240 \\
\hline & & $300 \mu \mathrm{g} / \mathrm{kg}$ & 4 & $336.5 \pm 36.0$ & 7.986 & \pm 0.322 & 1.611 & \pm 0.100 & $0.970 \pm 0.075$ & 2.017 & \pm 0.201 \\
\hline$\overline{\mathrm{EE}}$ & PND 1-5 & & 4 & $391.7 \pm 14.7$ & 7.908 & \pm 0 . & 1.533 & & $24 \pm 0$ & 1.947 & 140 \\
\hline & & $/ \mathrm{kg}$ & 3 & $367.0 \pm 22.8$ & 7.681 & \pm 0.568 & 1.477 & \pm 0.094 & $0.850 \pm 0.087$ & 1.548 & $\pm 0.136^{*}$ \\
\hline & & $100 \mu \mathrm{g} / \mathrm{kg}$ & 3 & $374.0 \pm 6.1$ & 6.920 & $\pm 0.110^{*}$ & 1.394 & \pm 0.112 & $0.700 \pm 0.096^{*}$ & 1.220 & $\pm 0.123^{* *}$ \\
\hline & PND 17-21 & Control & 3 & $353.9 \pm 32.7$ & 7.154 & \pm 0.245 & 1.456 & \pm 0.062 & $0.908 \pm 0.127$ & 1.978 & \pm 0.041 \\
\hline & & $10 \mu \mathrm{g} / \mathrm{kg}$ & 2 & $357.0 \pm 27.3$ & 7.201 & \pm 0.472 & 1.366 & \pm 0.099 & $1.013 \pm 0.143$ & 1.875 & \pm 0.179 \\
\hline & & $100 \mu \mathrm{g} / \mathrm{kg}$ & 3 & $373.1 \pm 21.3$ & 6.980 & \pm 0.316 & 1.521 & \pm 0.049 & $1.053 \pm 0.160$ & 2.118 & \pm 0.156 \\
\hline & PND 35-39 & Control & 4 & $357.4 \pm 26.0$ & 7.766 & \pm 0.479 & 1.567 & \pm 0.098 & $1.078 \pm 0.174$ & 1.954 & \pm 0.102 \\
\hline & & & 4 & $353.6 \pm 9.4$ & 7.586 & \pm 0.739 & 1.569 & \pm 0.131 & $0.960 \pm 0.084$ & 1.910 & \pm 0.236 \\
\hline & & $100 \mu \mathrm{g} / \mathrm{kg}$ & 4 & $347.1 \pm 27.6$ & 7.637 & \pm 0.700 & 1.517 & \pm 0.119 & $0.936 \pm 0.104$ & 1.783 & \pm 0.262 \\
\hline TAM & PND 1-5 & & 4 & $391.7 \pm 14.7$ & 7.908 & \pm 0.276 & 1.533 & \pm 0 . & $1.024 \pm 0$ & 1.947 & \pm 0.140 \\
\hline & & $\mathrm{mg} / \mathrm{kg}$ & 4 & $368.7 \pm 20.3$ & 7.153 & \pm 0.691 & 1.498 & \pm 0.084 & $0.813 \pm 0.120$ & 1.625 & \pm 0.271 \\
\hline & & $1 \mathrm{mg} / \mathrm{kg}$ & 4 & $356.4 \pm 22.4$ & 6.756 & \pm 1.014 & 1.454 & \pm 0.198 & $0.790 \pm 0.128 *$ & 1.601 & \pm 0.242 \\
\hline & & $3 \mathrm{mg} / \mathrm{kg}$ & 4 & $325.6 \pm 25.8 * *$ & 5.011 & $\pm 0.351 * *$ & 1.159 & $\pm 0.042 * *$ & $0.596 \pm 0.112 * *$ & 1.197 & $\pm 0.257^{* * *}$ \\
\hline & $\overline{\text { PND } 17-21}$ & Control & 4 & $357.5 \pm 26.3$ & 7.791 & \pm 0.654 & 1.432 & \pm 0.058 & $0.967 \pm 0.094$ & 1.903 & \pm 0.300 \\
\hline & & & 3 & $365.3 \pm 22.5$ & 7.788 & \pm 0.213 & 1.524 & \pm 0.155 & $0.935 \pm 0.178$ & 1.937 & \pm 0.160 \\
\hline & & $1 \mathrm{mg} / \mathrm{kg}$ & 4 & $351.9 \pm 31.7$ & 7.990 & \pm 0.766 & 1.541 & \pm 0.119 & $0.972 \pm 0.264$ & 1.871 & \pm 0.139 \\
\hline & & $3 \mathrm{mg} / \mathrm{kg}$ & 3 & $363.5 \pm 15.9$ & 7.457 & \pm 0.510 & 1.516 & \pm 0.095 & $0.927 \pm 0.131$ & 2.060 & \pm 0.090 \\
\hline & $\overline{\text { PND 35-39 }}$ & Control & 4 & $369.8 \pm 20.8$ & 7.711 & \pm 0.696 & 1.502 & \pm 0.053 & $0.895 \pm 0.041$ & 1.752 & \pm 0.115 \\
\hline & & $0.3 \mathrm{mg} / \mathrm{kg}$ & 3 & $348.8 \pm 37.5$ & 7.357 & \pm 0.352 & 1.548 & \pm 0.074 & $0.900 \pm 0.075$ & 1.733 & \pm 0.126 \\
\hline & & $1 \mathrm{mg} / \mathrm{kg}$ & 3 & $348.8 \pm 37.4$ & 8.189 & \pm 0.358 & 1.581 & \pm 0.136 & $0.959 \pm 0.079$ & 1.909 & \pm 0.235 \\
\hline & & $3 \mathrm{mg} / \mathrm{kg}$ & 3 & $339.8 \pm 10.0$ & 8.159 & \pm 0.212 & 1.626 & \pm 0.133 & $0.934 \pm 0.119$ & 1.866 & \pm 0.138 \\
\hline
\end{tabular}

Value: Mean \pm S.D. calculated using the litter as the unit.

FLU: flutamide; DDE: $p, p^{\prime}$-dichlorodiphenyldichloroethylene; VZ: vinclozolin; DES: diethylstilbestrol; EE: ethynylestradiol; TAM: tamoxifen; PND: postnatal day.

*: significantly different from control, $\mathrm{p}<0.05 ; * *$ : significantly different from control, $\mathrm{p}<0.01$. 
Table 7. Relative Organ Weights and Body Weights of PND 56 Male Rats Postnatally Exposed to Chemicals (Using the Individual Data)

\begin{tabular}{|c|c|c|c|c|c|c|c|c|}
\hline \multicolumn{2}{|c|}{$\begin{array}{l}\text { Chemical } \\
\text { Dosing period }\end{array}$} & Group & Males & $\begin{array}{l}\text { Body weight } \\
\text { (g) }\end{array}$ & $\begin{array}{l}\text { Testes } \\
(\mathrm{mg} / \mathrm{g})\end{array}$ & $\begin{array}{l}\text { Epididymides } \\
(\mathrm{mg} / \mathrm{g})\end{array}$ & $\begin{array}{l}\text { Ventral prostate } \\
(\mathrm{mg} / \mathrm{g})\end{array}$ & $\begin{array}{l}\text { Seminal Vesicles } \\
(\mathrm{mg} / \mathrm{g})\end{array}$ \\
\hline \multirow[t]{12}{*}{$\overline{\mathrm{FLU}}$} & PND 1-5 & Control & 15 & $383.1 \pm 24.2$ & $7.294 \pm 0.799$ & $1.397 \pm 0.105$ & $1.014 \pm 0.170$ & $1.986 \pm 0.304$ \\
\hline & & $1 \mathrm{mg} / \mathrm{kg}$ & 16 & $378.1 \pm 21.8$ & $7.536=0.528$ & $1.511 \pm 0.130$ & $0.986 \pm 0.194$ & $1.895 \pm 0.255$ \\
\hline & & $10 \mathrm{mg} / \mathrm{kg}$ & 16 & $389.8 \pm 34.8$ & $7.441 \pm 0.751$ & $1.443 \pm 0.111$ & $0.998 \pm 0.146$ & $1.774 \pm 0.249$ \\
\hline & & $30 \mathrm{mg} / \mathrm{kg}$ & 15 & $371.0 \pm 27.8$ & $7.574 \pm 0.684$ & $1.431 \pm 0.107$ & $1.049 \pm 0.112$ & $1.801 \pm 0.276$ \\
\hline & $\overline{\text { PND } 17-21}$ & Control & 12 & $376.2 \pm 15.8$ & $7.446 \pm 0.542$ & $1.438 \pm 0.124$ & $0.989 \pm 0.124$ & $1.758 \pm 0.292$ \\
\hline & & $1 \mathrm{mg} / \mathrm{kg}$ & 11 & $359.1 \pm 21.3$ & $7.388 \pm 0.609$ & $1.511 \pm 0.205$ & $1.016 \pm 0.132$ & $1.862 \pm 0.307$ \\
\hline & & $10 \mathrm{mg} / \mathrm{kg}$ & 12 & $372.1 \pm 27.8$ & $7.508 \pm 0.590$ & $1.451 \pm 0.084$ & $0.951 \pm 0.185$ & $1.918 \pm 0.170$ \\
\hline & & $30 \mathrm{mg} / \mathrm{kg}$ & 12 & $376.4 \pm 20.4$ & $7.707 \pm 0.428$ & $1.484 \pm 0.100$ & $1.043 \pm 0.127$ & $1.923 \pm 0.388$ \\
\hline & PND 35-39 & Control & 16 & $377.3 \pm 33.2$ & $7.654 \pm 0.377$ & $1.520 \pm 0.067$ & $1.068 \pm 0.094$ & $2.065 \pm 0.238$ \\
\hline & & $1 \mathrm{mg} / \mathrm{kg}$ & 16 & $382.8 \pm 26.3$ & $8.108 \pm 0.811$ & $1.603 \pm 0.165$ & $1.097 \pm 0.172$ & $2.108 \pm 0.301$ \\
\hline & & $10 \mathrm{mg} / \mathrm{kg}$ & 15 & $378.4 \pm 26.7$ & $7.778 \pm 0.859$ & $1.411+0.139$ & $1.053 \pm 0.192$ & $1.755 \pm 0.324^{*}$ \\
\hline & & $30 \mathrm{mg} / \mathrm{kg}$ & 16 & $381.1 \pm 22.1$ & $7.951 \pm 0.487$ & $1.398 \pm 0.114^{*}$ & $0.866 \pm 0.154 * *$ & $1.788 \pm 0.266^{*}$ \\
\hline$\overline{\mathrm{DDE}}$ & PND 1-5 & Control & 12 & $362.5 \pm 14.6$ & $7.760 \pm 0.369$ & $1.447 \pm 0.090$ & $0.809 \pm 0.130$ & $1.942 \pm 0.267$ \\
\hline & & $10 \mathrm{mg} / \mathrm{kg}$ & 12 & $382.2 \pm 32.2$ & $7.122 \pm 0.498^{*}$ & $1.395 \pm 0.108$ & $0.852 \pm 0.169$ & $1.874 \pm 0.226$ \\
\hline & & $30 \mathrm{mg} / \mathrm{kg}$ & 12 & $367.2 \pm 26.8$ & $7.670 \pm 0.442$ & $1.443 \pm 0.102$ & $0.928 \pm 0.112$ & $1.893 \pm 0.292$ \\
\hline & & $100 \mathrm{mg} / \mathrm{kg}$ & 11 & $367.9 \pm 28.3$ & $7.460 \pm 0.544$ & $1.422 \pm 0.074$ & $0.826 \pm 0.098$ & $1.789 \pm 0.214$ \\
\hline & & $300 \mathrm{mg} / \mathrm{kg}$ & 12 & $374.0 \pm 26.9$ & $8.024 \pm 0.658$ & $1.398 \pm 0.096$ & $0.749 \pm 0.084$ & $1.783 \pm 0.288$ \\
\hline & $\overline{\text { PND } 17-21}$ & Control & 12 & $358.6 \pm 24.0$ & $8.522=1.002$ & $1.539 \pm 0.142$ & $0.815 \pm 0.171$ & $1.724 \pm 0.334$ \\
\hline & & $10 \mathrm{mg} / \mathrm{kg}$ & 12 & $377.0 \pm 25.9$ & $7.804=0.660$ & $1.465 \pm 0.131$ & $0.893 \pm 0.077$ & $1.830 \pm 0.308$ \\
\hline & & $30 \mathrm{mg} / \mathrm{kg}$ & 11 & $358.8 \pm 24.7$ & $7.830 \pm 0.568$ & $1.489 \pm 0.109$ & $0.865 \pm 0.095$ & $1.676 \pm 0.174$ \\
\hline & & $100 \mathrm{mg} / \mathrm{kg}$ & 12 & $362.8 \pm 18.3$ & $8.263 \pm 0.382$ & $1.502 \pm 0.064$ & $0.840 \pm 0.103$ & $1.751 \pm 0.211$ \\
\hline & & $300 \mathrm{mg} / \mathrm{kg}$ & 13 & $359.1 \pm 38.0$ & $8.731 \pm 0.864$ & $1.430 \pm 0.124$ & $0.841 \pm 0.159$ & $1.558 \pm 0.178$ \\
\hline & PND 35-39 & Control & 12 & $333.5 \pm 26.1$ & $7.844 \pm 0.731$ & $1.520 \pm 0.124$ & $0.925 \pm 0.209$ & $1.834 \pm 0.331$ \\
\hline & & $10 \mathrm{mg} / \mathrm{kg}$ & 12 & $356.0 \pm 24.1$ & $7.362 \pm 0.634$ & $1.323 \pm 0.112^{* *}$ & $0.875 \pm 0.084$ & $1.831 \pm 0.153$ \\
\hline & & $30 \mathrm{mg} / \mathrm{kg}$ & 12 & $347.2 \pm 41.8$ & $7.500=0.372$ & $1.376 \pm 0.069^{*}$ & $0.908 \pm 0.097$ & $1.807 \pm 0.238$ \\
\hline & & $100 \mathrm{mg} / \mathrm{kg}$ & 12 & $353.5 \pm 26.9$ & $7.823 \pm 0.492$ & $1.409 \pm 0.156$ & $0.847 \pm 0.119$ & $1.523 \pm 0.247^{* *}$ \\
\hline$\overline{\mathrm{VZ}}$ & PND 1-5 & Control & 15 & $368.9 \pm 30.7$ & $7.917 \pm 1.053$ & $1.590 \pm 0.158$ & $1.039 \pm 0.153$ & $2.168 \pm 0.277$ \\
\hline & & $\mathrm{g} / \mathrm{kg}$ & 12 & $382.0 \pm 35.0$ & $7.392 \pm 0.688$ & $1.477 \pm 0.100$ & $1.006 \pm 0.137$ & $2.059 \pm 0.160$ \\
\hline & & $30 \mathrm{mg} / \mathrm{kg}$ & 15 & $379.6 \pm 16.8$ & $8.020 \pm 0.761$ & $1.505 \pm 0.136$ & $0.917 \pm 0.130$ & $2.048 \pm 0.302$ \\
\hline & & $100 \mathrm{mg} / \mathrm{kg}$ & 15 & $376.3 \pm 24.8$ & $7.361 \pm 1.030$ & $1.589 \pm 0.134$ & $1.025 \pm 0.187$ & $2.115 \pm 0.195$ \\
\hline & $\overline{P N D} 17-21$ & Control & 16 & $357.5 \pm 28.1$ & $7.791 \pm 0.655$ & $1.432 \pm 0.086$ & $0.967 \pm 0.133$ & $1.903 \pm 0.317$ \\
\hline & & $10 \mathrm{mg} / \mathrm{kg}$ & 16 & $366.8 \pm 24.6$ & $7.981=0.658$ & $1.562 \pm 0.126$ & $0.987 \pm 0.101$ & $2.041 \pm 0.302$ \\
\hline & & $30 \mathrm{mg} / \mathrm{kg}$ & 16 & $378.3 \pm 26.0$ & $7.629 \pm 0.831$ & $1.550 \pm 0.182$ & $0.891 \pm 0.117$ & $1.931 \pm 0.287$ \\
\hline & & $100 \mathrm{mg} / \mathrm{kg}$ & 15 & $373.2 \pm 30.3$ & $8.056 \pm 0.710$ & $1.527 \pm 0.146$ & $1.022 \pm 0.156$ & $2.117 \pm 0.277$ \\
\hline & PND $35-39$ & Control & 16 & $369.8 \pm 25.8$ & $7.711 \pm 0.746$ & $1.502 \pm 0.098$ & $0.895 \pm 0.153$ & $1.752 \pm 0.190$ \\
\hline & & $10 \mathrm{mg} / \mathrm{kg}$ & 12 & $373.3 \pm 35.7$ & $7.420 \pm 0.647$ & $1.572 \pm 0.122$ & $0.910 \pm 0.100$ & $1.911 \pm 0.231$ \\
\hline & & $30 \mathrm{mg} / \mathrm{kg}$ & 12 & $361.7 \pm 31.7$ & $8.125=0.562$ & $1.546 \pm 0.076$ & $0.982 \pm 0.114$ & $1.885 \pm 0.154$ \\
\hline & & $100 \mathrm{mg} / \mathrm{kg}$ & 12 & $363.1 \pm 14.2$ & $7.719 \pm 0.393$ & $1.508 \pm 0.134$ & $0.941 \pm 0.132$ & $1.907 \pm 0.203$ \\
\hline$\overline{\text { DES }}$ & PND 1-5 & Control & 16 & $367.5 \pm 31.8$ & $8.089 \pm 0.914$ & $1.560 \pm 0.174$ & $1.064 \pm 0.155$ & $1.948 \pm 0.262$ \\
\hline & & $10 \mu \mathrm{g} / \mathrm{kg}$ & 16 & $365.7 \pm 26.6$ & $7.296 \pm 0.829$ & $1.391 \pm 0.152 *$ & $0.917 \pm 0.167^{*}$ & $1.870 \pm 0.390$ \\
\hline & & $100 \mu \mathrm{g} / \mathrm{kg}$ & 16 & $383.6 \pm 23.0$ & $8.277 \pm 2.095$ & $1.431 \pm 0.127$ & $0.769 \pm 0.129^{* *}$ & $1.668 \pm 0.527$ \\
\hline & & $300 \mu \mathrm{g} / \mathrm{kg}$ & 15 & $343.0 \pm 38.6$ & $7.893 \pm 2.723$ & $1.569 \pm 0.271$ & $0.835 \pm 0.120 * *$ & $1.321 \pm 0.232^{* *}$ \\
\hline & $\overline{P N D} 17-21$ & Control & 15 & $340.4 \pm 35.0$ & $7.783 \pm 0.497$ & $1.566 \pm 0.156$ & $0.927 \pm 0.097$ & $1.962 \pm 0.353$ \\
\hline & & $10 \mu \mathrm{g} / \mathrm{kg}$ & 12 & $337.2 \pm 26.7$ & $7.906 \pm 0.674$ & $1.432 \pm 0.105^{*}$ & $0.922 \pm 0.132$ & $1.781 \pm 0.209$ \\
\hline & & $100 \mu \mathrm{g} / \mathrm{kg}$ & 16 & $348.9 \pm 24.8$ & $6.940 \pm 0.743^{* *}$ & $1.455 \pm 0.118$ & $0.900 \pm 0.109$ & $1.939 \pm 0.283$ \\
\hline & & $300 \mu \mathrm{g} / \mathrm{kg}$ & 16 & $352.8 \pm 20.5$ & $7.309 \pm 0.846$ & $1.417 \pm 0.121 * *$ & $0.933 \pm 0.117$ & $1.935 \pm 0.346$ \\
\hline & PND 35-39 & Control & 15 & $360.3 \pm 36.2$ & $7.793 \pm 0.609$ & $1.539 \pm 0.168$ & $1.052 \pm 0.194$ & $2.299 \pm 0.488$ \\
\hline & & $10 \mu \mathrm{g} / \mathrm{kg}$ & 12 & $344.9 \pm 39.7$ & $7.472 \pm 0.686$ & $1.593 \pm 0.155$ & $1.035 \pm 0.140$ & $1.963 \pm 0.240$ \\
\hline & & $100 \mu \mathrm{g} / \mathrm{kg}$ & 16 & $330.4 \pm 26.3$ & $7.920=0.736$ & $1.556 \pm 0.089$ & $0.987 \pm 0.193$ & $1.910 \pm 0.351$ \\
\hline & & $300 \mu \mathrm{g} / \mathrm{kg}$ & 16 & $336.5 \pm 36.5$ & $7.986 \pm 0.777$ & $1.611 \pm 0.153$ & $0.970 \pm 0.134$ & $2.017 \pm 0.260$ \\
\hline$\overline{\mathrm{EE}}$ & PND 1-5 & Control & 16 & $391.7 \pm 17.3$ & $7.908 \pm 0.437$ & $1.533 \pm 0.131$ & $1.024 \pm 0.128$ & $1.947 \pm 0.269$ \\
\hline & & $10 \mu \mathrm{g} / \mathrm{kg}$ & 12 & $367.0 \pm 22.9^{* *}$ & $7.681 \pm 0.690$ & $1.477 \pm 0.103$ & $0.850 \pm 0.110^{* *}$ & $1.548 \pm 0.270^{*}$ \\
\hline & & $100 \mu \mathrm{g} / \mathrm{kg}$ & 10 & $372.7 \pm 15.4^{*}$ & $6.919 \pm 0.402 * *$ & $1.387 \pm 0.149 *$ & $0.692 \pm 0.114^{* *}$ & $1.223 \pm 0.166^{* *}$ \\
\hline & $\overline{\text { PND } 17-21}$ & Control & 12 & $353.9 \pm 32.3$ & $7.154 \pm 0.561$ & $1.456 \pm 0.126$ & $0.908 \pm 0.147$ & $1.978 \pm 0.207$ \\
\hline & & $10 \mu \mathrm{g} / \mathrm{kg}$ & 8 & $357.0 \pm 27.4$ & $7.201 \pm 0.538$ & $1.366 \pm 0.101$ & $1.013 \pm 0.149$ & $1.875 \pm 0.277$ \\
\hline & & $100 \mu \mathrm{g} / \mathrm{kg}$ & 12 & $373.1 \pm 23.1$ & $6.980 \pm 0.530$ & $1.521 \pm 0.101$ & $1.053 \pm 0.187$ & $2.118 \pm 0.245$ \\
\hline & $\overline{\text { PND 35-39 }}$ & Control & 16 & $357.4 \pm 29.4$ & $7.766 \pm 0.783$ & $1.567 \pm 0.118$ & $1.078 \pm 0.196$ & $1.954 \pm 0.286$ \\
\hline & & $10 \mu \mathrm{g} / \mathrm{kg}$ & 16 & $353.6 \pm 17.8$ & $7.586 \pm 0.738$ & $1.569 \pm 0.149$ & $0.960 \pm 0.147$ & $1.910 \pm 0.347$ \\
\hline & & $100 \mu \mathrm{g} / \mathrm{kg}$ & 16 & $347.1 \pm 31.1$ & $7.637 \pm 0.870$ & $1.517 \pm 0.144$ & $0.936 \pm 0.123 *$ & $1.783 \pm 0.267$ \\
\hline TAM & PND 1-5 & Control & 16 & $391.7 \pm 17.3$ & $7.908 \pm 0.437$ & $1.533 \pm 0.131$ & $1.024 \pm 0.128$ & $1.947 \pm 0.269$ \\
\hline & & $0.3 \mathrm{mg} / \mathrm{kg}$ & 16 & $368.7 \pm 27.9^{*}$ & $7.153 \pm 0.772$ & $1.498 \pm 0.115$ & $0.813 \pm 0.161 * *$ & $1.625 \pm 0.352^{*}$ \\
\hline & & $1 \mathrm{mg} / \mathrm{kg}$ & 16 & $356.4 \pm 28.8 * *$ & $6.756 \pm 1.135 *$ & $1.454 \pm 0.219$ & $0.790 \pm 0.181^{* *}$ & $1.601 \pm 0.403 *$ \\
\hline & & $3 \mathrm{mg} / \mathrm{kg}$ & 15 & $327.9 \pm 29.0^{* *}$ & $5.043 \pm 0.550^{* *}$ & $1.162 \pm 0.114^{* *}$ & $0.606 \pm 0.124 * *$ & $1.218 \pm 0.371^{* *}$ \\
\hline & PND 17-21 & Control & 16 & $357.5 \pm 28.1$ & $7.791 \pm 0.655$ & $1.432 \pm 0.086$ & $0.967 \pm 0.133$ & $1.903 \pm 0.317$ \\
\hline & & $0.3 \mathrm{mg} / \mathrm{kg}$ & 11 & $364.5 \pm 29.6$ & $7.768 \pm 0.452$ & $1.530 \pm 0.189$ & $0.950 \pm 0.196$ & $1.936 \pm 0.244$ \\
\hline & & $1 \mathrm{mg} / \mathrm{kg}$ & 15 & $354.0 \pm 31.8$ & $7.913 \pm 0.812$ & $1.530 \pm 0.126$ & $0.945 \pm 0.243$ & $1.873 \pm 0.236$ \\
\hline & & $3 \mathrm{mg} / \mathrm{kg}$ & 12 & $363.5 \pm 21.1$ & $7.457 \pm 0.901$ & $1.516 \pm 0.121$ & $0.927 \pm 0.212$ & $2.060 \pm 0.374$ \\
\hline & PND $35-39$ & Control & 16 & $369.8 \pm 25.8$ & $7.711 \pm 0.746$ & $1.502 \pm 0.098$ & $0.895 \pm 0.153$ & $1.752 \pm 0.190$ \\
\hline & & $0.3 \mathrm{mg} / \mathrm{kg}$ & 12 & $348.8 \pm 37.5$ & $7.357 \pm 0.457$ & $1.548 \pm 0.095$ & $0.900 \pm 0.102$ & $1.733 \pm 0.173$ \\
\hline & & $1 \mathrm{mg} / \mathrm{kg}$ & 12 & $348.8 \pm 42.4$ & $8.189 \pm 0.787$ & $1.581 \pm 0.134$ & $0.959 \pm 0.141$ & $1.909 \pm 0.291$ \\
\hline & & $3 \mathrm{mg} / \mathrm{kg}$ & 12 & $339.8 \pm 16.1^{*}$ & $8.159 \pm 0.596$ & $1.626 \pm 0.134 *$ & $0.934 \pm 0.139$ & $1.866 \pm 0.194$ \\
\hline
\end{tabular}

Value: Mean \pm S.D. calculated using the individual data.

FLU: flutamide; DDE: $p, p^{\prime}$-dichlorodiphenyldichloroethylene; VZ: vinclozolin; DES: diethylstilbestrol; EE: ethynylestradiol; TAM: tamoxifen; PND: postnatal day; *: significantly different from control, $\mathrm{p}<0.05$; **: significantly different from control, $\mathrm{p}<0.01$. 

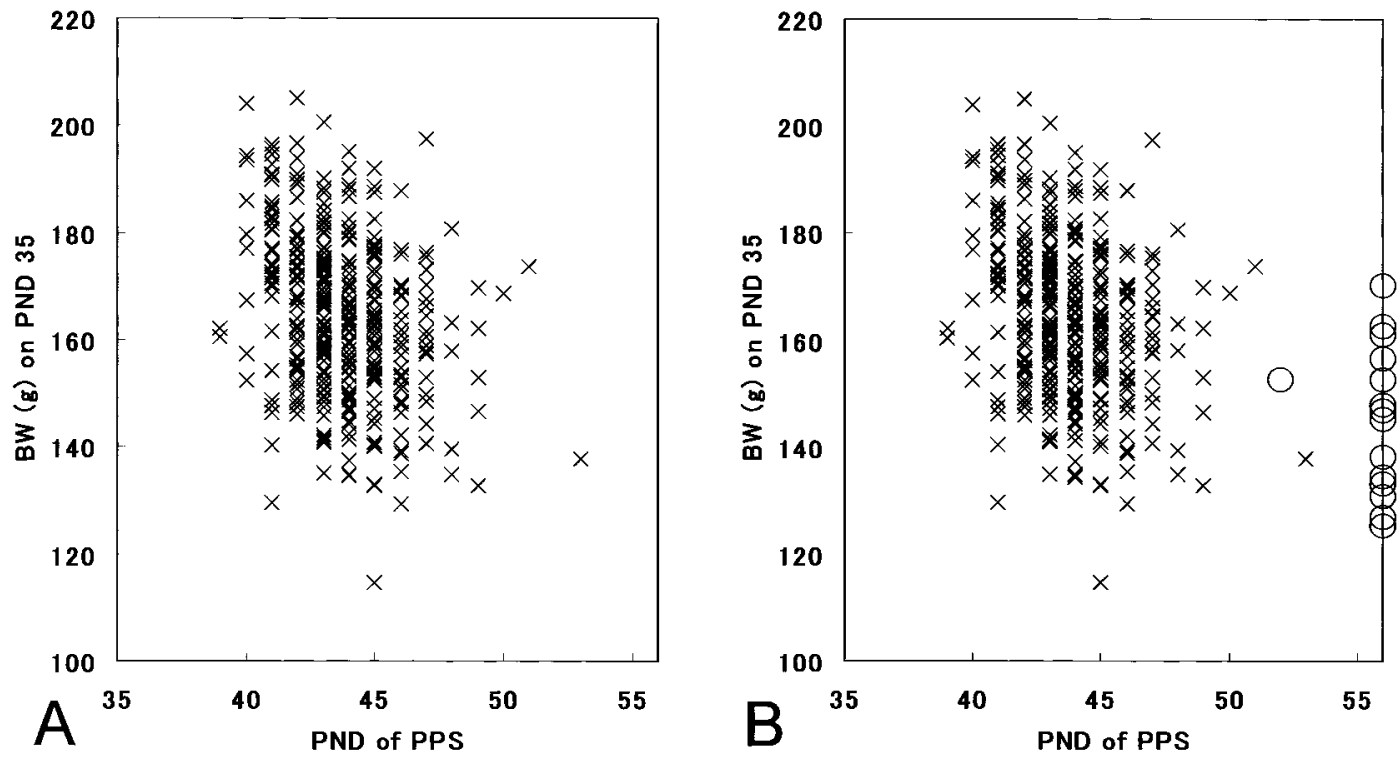

Fig. 5. Correlation diagram between the day of preputial separation and body weight of control and dosed rats.

A: There is a negative correlation between PND of preputial separation (PPS) and body weight on PND 35 . Number of cases $=366$, Pearson's correlation coefficient: $\mathrm{r}=-0.30$. B: Preputial separation (PPS) of males exposed to $3 \mathrm{mg} / \mathrm{kg}$ tamoxifen on PND 1-5 is delayed and the data are plotted as "O" outside the range of control data " $\mathrm{X}$ ".

\section{3) Pathological examination}

Unilateral or bilateral cloudy white discoloration of the efferent ductule as well as testis enlargement was increased in the groups exposed to EE and DES on PND 1-5. Histopathological examination revealed retention of sperm or inflammatory cells in the lumen of the efferent ductule and edema in adipose tissue surrounding the dilated ductule. Organs of the EE or TAM groups showed no remarkable histological changes except for a relative atrophic appearance.

\section{Correlation between preputial separation and body weight}

The control animal data in this study $(n=366)$ are symbolized by " $X$ " on the scattergram of Fig. 5A. PND of preputial separation and body weight on PND 35 showed a negative correlation (Pearson's correlation coefficient: $\mathrm{r}=$ -0.30 ). The data from animals treated with $3 \mathrm{mg} / \mathrm{kg}$ TAM, symbolized by "O", are compared in Fig. 5B. Body weight on PND 35 in this group showed significant reduction. Preputial separation of TAM treated males was delayed, and the data were outside the range of the control data.

\section{Discussion}

Preputial separation in untreated rats initiates from cornification of the epithelium lying between the glans penis and prepuce ${ }^{1,2}$. The cornification progresses from the tip of the glans penis towards its base and from the dorsal surface to ventral aspect of the glans penis. Preputial separation is considered complete when cornification reaches the ventral end of the glans penis. However, complete separation was not observed in animals exposed to FLU or VZ in their fetal period, since they had a cleft phallus and hypospadias. Induction of hypospadias is reportedly caused by FLU, VZ and finasteride $\mathrm{e}^{914-19}$. FLU, a well-known potent androgen receptor antagonist, is used as a non-steroidal, anti-androgen drug for the treatment of prostate cancer. FLU inhibits TS and DHT binding to the intracellular androgen receptor, and prenatal/perinatal FLU exposure induces abnormalities in the genital tract of rats such as hypospadias and agenesis of the prostate, epididymis, and vas deferens $s^{9,14,15}$. The fungicide, VZ, is also an androgen receptor antagonist. It induces hypospadias in rats after perinatal or prenatal administration $^{16,17}$. Finasteride, which inhibits $5 \alpha$-reductase conversion of TS to DHT, also induces hypospadias in male rats exposed to it from GD 15 to day 21 postpartum ${ }^{18}$ or GD $6-20^{19}$, and based on this finding, DHT is thought to be involved in the development of the external genitalia.

The most sensitive period to induce hypospadias is reportedly GD $15-16$ with $400 \mathrm{mg} / \mathrm{kg}$ of $\mathrm{VZ}^{17}$, while only weak sensitivity was found with treatment on GD 17-18. Finasteride-exposed rats also showed similar results ${ }^{18}$. In our present study, exposure to $100 \mathrm{mg} / \mathrm{kg}$ of VZ on GD 1417 induced hypospadias and a cleft phallus with cleft prepuce, but exposure on GD 18-21 did not induce any abnormalities in the external genitalia. At a higher dose of $200 \mathrm{mg} / \mathrm{kg}$, VZ caused the death of the pregnant females or newborn pups (data not shown). Exposure to $10 \mathrm{mg} / \mathrm{kg}$ of FLU on GD 14-17 induced hypospadias with a cleft phallus and cleft prepuce, while exposure on GD 18-21 induced hypospadias and a cleft phallus without a cleft prepuce. 
Higher doses of FLU induced the same abnormality as our previous study ${ }^{2}$. Although both FLU and VZ are androgen receptor antagonists, their sensitive periods differed: cleft phallus was caused by FLU administration until later in pregnancy. FLU exposed males without the malformation showed a hypoplastic penis and a delay in preputial separation, but prenatal exposure to other chemicals did not affect the preputial separation.

Postnatal chemical exposure influenced preputial separation in a variety of ways. Anti-androgen, FLU caused a delay when administered on PND 35-39, and statistical analyses using individual data of DDE and VZ also showed significant delays of preputial separation, but neonatal exposure to these chemicals on PND 1-5 did not influence the time of preputial separation. On the other hand, neonatal administration of EE and TAM induced a marked delay or incomplete separation. DES exposure during both PND 1-5 and PND 35-39 caused a delay in separation. Preputial separation is thought to be dependent on the continued presence of androgen after PND 35, since castration on PND 35 blocks preputial separation and the addition of TS or DHT reverses the effects of castration ${ }^{3}$. Male rat serum testosterone levels have been reported to decrease after birth and to increase at the prepubertal stage ${ }^{11}$. Testosterone during fetal life is thought to act to masculinize the genitalia, and testosterone at puberty may act on the maturation of the target organ. Prenatal exposure to the anti-androgens used in the present study resulted in malformation of the external genitalia, and the effect of their prepubertal exposure was a delay in preputial separation. These results may indicate that anti-androgens effect on male rats in a relatively higher level of serum testosterone, and that male rats are not sensitive to neonatal exposure to anti-androgenic chemicals.

Neonatal exposure of estrogenic chemicals is known to induce marked effects on male rats. Delay of preputial separation and decrease of testis and prostate weight with a reduction of plasma testosterone levels have been reported in rats neonatally administered estradiol benzoate ${ }^{20}$. Neonatal treatment of DES or EE has also been reported to cause dosedependent reductions in plasma testosterone levels and testis weights in adulthood ${ }^{21}$. An estrogen receptor is found in the male reproductive tract ${ }^{22,23}$. These findings suggest that estrogen is relevant to the growth of the male reproductive organs. The delay of preputial separation induced by DES, EE and TAM exposures on PND 1-5 in the present study is thought to be caused by estrogen-related effects, and the delay may have not only been caused by the direct effects on the genital tract but also by effects on the systemic endocrine function. Prepubertal exposure to DES induced a delay of preputial separation in male rats in the present study, the same as observed for anti-androgenic chemicals. Serum testosterone levels in adult or prepubertal male rats have been reported to be increased by $\mathrm{FLU}^{24,25}, \mathrm{DDE}^{5}$ and $\mathrm{VZ}^{24}$ treatment, and the increase is thought to be caused by their anti-androgenic effects. It has been reported that serum testosterone levels in adult male rats treated with DES are decreased $^{26}$, thus, testosterone reduction may be the reason for the delay of preputial separation seen in the DES group prepubertally treated in our present study. EE and TAM have also been reported to decrease serum testosterone levels ${ }^{25,27}$, but these chemicals did not delay preputial separation in our present study. The reason for the different effects of DES and EE/TAM treatments were not revealed in our study.

A negative correlation between the body weight on PND 35 and the day of preputial separation was demonstrated in the control males (Fig. 5A). This diagram shows a tendency for males of higher body weight to complete preputial separation earlier than males of lower body weight. Ashby and Lefevre ${ }^{8}$ thought that there was a marked dependence of the day of preputial separation on the initial body weight of the test animals, and that delays in preputial separation can only be interpreted with confidence when they are not accompanied by losses in body weight. In our present study, males exposed to $3 \mathrm{mg} / \mathrm{kg}$ TAM on PND 1-5 showed significantly lower body weight on PND 35, and their preputial separation was delayed. The delay may not depend on the reduced weight gain, since 14 males out of 15 did not show complete separation of the prepuce on the day of autopsy, PND 56, and their data were outside the range of control data.

AGD was reduced by FLU and VZ exposures on GD 14-17. Reduction of AGD by anti-androgen has been reported, and AGD is thought to have high sensitivity to antiandorogens ${ }^{17}$. In the present study, however, there were no apparent changes in AGD after DDE exposure. DES also caused a decrease in AGD with GD 18-21 exposure, and there was a difference between the results of DES and EE/ TAM exposure. Histological examination revealed a tortuous and bent cavernous body of the penis seen in the sagittal section of males prenatally exposed to FLU or VZ and sacrificed on PND 6. This morphological change may be a reason for the reduction in AGD.

The relative weights of the ventral prostate in males prenatally exposed to $\mathrm{VZ}$ or DES were decreased significantly. In these groups, prostate aplasia or hypoplasia was observed in the group exposed to VZ on GD 14-17 and DES on GD 18-21. Postnatal FLU exposure decreased the relative weight of the ventral prostate in the group dosed on PND 35-39. No apparent effect was detected in males postnatally exposed to DDE or VZ. PND 1-5 exposure of EE or TAM induced a reduction in the weight of the testis and other reproductive organs. Although the statistical analyses using the individual data showed significant results in the lower dose group and the other organs, there were no significant differences in the groups exposed to VZ. These findings indicate that preputial separation is more useful than the measurement of organ weight as an endpoint in detecting endocrine active chemicals under the conditions used in this study.

Prenatal exposure of anti-androgens FLU and VZ induced hypospadias, and the time of preputial separation could not be determined. Although GD 18-21 exposure was expected to produce a very low incidence of hypospadias, 
FLU exposure on GD 18-21 induced the abnormality. Other chemicals did not induce hypospadias or delays of the preputial separation. From these results, it is unclear if preputial separation after prenatal exposure is a useful way for detecting endocrine active chemicals. On the other hand, prepubertal exposure to FLU, DDE and VZ caused delays in preputial separation, and neonatal exposure to EE and TAM induced delays in separation with a reduction in organ and body weight gain. Both neonatal and prepubertal exposure to DES caused a delay in preputial separation. These results indicate that neonatal (PND 1-10) and prepubertal exposure may be useful for detecting endocrine active chemicals by observing preputial separation, and that continuous administration of chemicals from PND 35 to the day of preputial separation may be more effective for prepubertal exposure.

In conclusion, the usability of preputial separation to detect endocrine active chemicals after prenatal exposure to them is still unclear. Postnatal exposure, however, may be a useful method for a screening assay to detect endocrine active chemicals by preputial separation, and postnatal exposure is dependent on both neonatal (PND 1-10) and prepubertal continuous exposure.

Acknowledgements: This work was supported by Health and Labour Sciences Research Grants from the Ministry of Health, Labour and Welfare of Japan.

\section{References}

1. Lyons WR, Berlin I, and Friedlander S. Cornification of balano-preputial epithelium in normal rats and in castrated rats treated with testosterone propionate. Endocrinology. 31: 659-663. 1942.

2. Yoshimura S, Yamaguchi H, Konno K, Ohsawa N, Noguchi $\mathrm{S}$, and Chisaka A. Hypospadias and incomplete preputial separation in male rats induced by prenatal exposure to an anti-androgen, flutamide. J Toxicol Pathol. 17: 113-118. 2004.

3. Korenbrot CC, Huhtaniemi IT, and Weiner RI. Preputial separation as an external sign of pubertal development in the male rat. Biol Reprod. 17: 298-303. 1977.

4. Yamada T, Sunami O, Kunimatsu T, Kamita Y, Okuno Y, Seki T, Nakatsuka I, and Matsuo M. Dissection and weighing of accessory sex glands after formalin fixation, and a 5-day assay using young mature rats are reliable and feasible in the Hershberger assay. Toxicology. 162: 103119. 2001

5. O'Connor JC, Frame SR, Davis LG, and Cook JC. Detection of the environmental antiandrogen $p, p^{\prime}$-DDE in CD and Long-Evans rats using a tier I screening battery and a Hershberger assay. Toxicol Sci. 51: 44-53. 1999.

6. Sunami O, Kunimatsu T, Yamada T, Yabushita S, Sukata T, Miyata K, Kamita Y, Okuno Y, Seki T, Nakatsuka I, and Matsuo M. Evaluation of a 5-day Hershberger assay using young mature male rats: methyltestosterone and $p, p^{\prime}$-DDE, but not fenitrothion, exhibited androgenic or antiandrogenic activity in vivo. J Toxicol Sci. 25: 403-415. 2000.

7. Yamasaki K, Sawaki M, Ohta R, Okuda H, Katayama S,
Yamada T, Ohta T, Kosaka T, and Owens W. OECD validation of the Hershberger assay in Japan: phase 2 dose response of methyltestosterone, vinclozolin, and $p, p^{\prime}$-DDE. Environ Health Perspect. 111: 1912-1919. 2003.

8. Ashby $\mathrm{J}$ and Lefevre PA. The peripubertal male rat assay as an alternative to the Hershberger castrated male rat assay for the detection of anti-androgens, oestrogens and metabolic modulators. J Appl Toxicol. 20: 35-47. 2000.

9. McIntyre BS, Barlow NJ, and Foster PMD. Androgenmediated development in male rat offspring exposed to flutamide in utero: permanence and correlation of early postnatal changes in anogenital distance and nipple retention with malformations in androgen-dependent tissues. Toxicol Sci. 62: 236-249. 2001.

10. Gaytan F, Pinilla L, Romero JL, and Aguilar E. Differential effects of the administration of human chorionic gonadotropin to postnatal rats. J Endocrinol. 142: 527-534. 1994.

11. Paz GF, Winter JSD, Reyes FI, and Faiman C. Developmental pattern of testosterone production by the rat testis. Steroids. 36: 675-688. 1980.

12. Watanabe C, Kuwagata M, Yoshimura S, Azegami J, Kojima K, Ono H, and Nagao T. An improved technique for repeated gavage administration to rat neonates. Congenit Anom (Kyoto). 43: 177-179. 2003.

13. Wada K. Method and problem in measurement of AGD and preputial separation (in Japanese). Annual Report of Hatano Research Institute. 25: 109-114. 2002.

14. Mylchreest E, Sar M, Cattley RC, and Foster PMD. Disruption of androgen-regulated male reproductive development by di(n-butyl) phthalate during late gestation in rats is different from flutamide. Toxicol Appl Pharmacol. 156: 81-95. 1999.

15. Miyata K, Yabushita S, Sukata T, Sano M, Yoshino H, Nakanishi T, Okuno Y, and Matsuo M. Effects of perinatal exposure to flutamide on sex hormones and androgendependent organs in F1 male rats. J Toxicol Sci. 27: 19-33. 2002.

16. Gray LE Jr, Ostby JS, and Kelce WR. Developmental effects of an environmental antiandrogen: the fungicide vinclozolin alters sex differentiation of the male rat. Toxicol Appl Pharmacol. 129: 46-52. 1994.

17. Wolf CJ, LeBlanc GA, Ostby JS, and Gray LE Jr. Characterization of the period of sensitivity of fetal male sexual development to vinclozolin. Toxicol Sci. 55: 152161. 2000.

18. Clark RL, Anderson CA, Prahalada S, Robertson RT, Lochry EA, Leonard YM, Stevens JL, and Hoberman AM. Critical developmental periods for effects on male rat genitalia induced by finasteride, a $5 \alpha$-reductase inhibitor. Toxicol Appl Pharmacol. 119: 34-40. 1993.

19. Anderson CA and Clark RL. External genitalia of the rat: normal development and the histogenesis of $5 \alpha$-reductase inhibitor-induced abnormalities. Teratology. 42: 483-496. 1990.

20. Bellido C, Gaytan F, Aguilar R, Pinilla L, and Aguilar E. Prepuberal reproductive defects in neonatal estrogenized male rats. Biol Reprod. 33: 381-387. 1985.

21. Atanassova N, McKinnell C, Walker M, Turner KJ, Fisher JS, Morley M, Millar MR, Groome NP, and Sharpe RM. Permanent effects of neonatal estrogen exposure in rats on reproductive hormone levels, Sertoli cell number, and the 
efficiency of spermatogenesis in adulthood. Endocrinology. 140: 5364-5373. 1999.

22. Fisher JS, Millar MR, Majdic G, Saunders PT, Fraser HM, and Sharpe RM. Immunolocalisation of oestrogen receptor$\alpha$ within the testis and excurrent ducts of the rat and marmoset monkey from perinatal life to adulthood. J Endocrinol. 153: 485-495. 1997.

23. Saunders PTK, Fisher JS, Sharpe RM, and Millar MR. Expression of oestrogen receptor beta (ER $\beta$ ) occurs in multiple cell types, including some germ cells, in the rat testis. J Endocrinol. 156: R13-17. 1998.

24. Yu WJ, Lee BJ, Nam SY, Ahn B, Hong JT, Do JC, Kim YC, Lee YS, and Yun YW. Reproductive disorders in pubertal and adult phase of the male rats exposed to vinclozolin during puberty. J Vet Med Sci. 66: 847-853. 2004.

25. Okuno $\mathrm{Y}$ and Miyata K. Effects of an endocrine disruptor on male reproductive organs. J Toxicol Pathol. 14: 79-82. 2001.

26. Goyal HO, Braden TD, Mansour M, Williams CS, Kamaleldin A, and Srivastava KK. Diethylstilbestrol-treated adult rats with altered epididymal sperm numbers and sperm motility parameters, but without alterations in sperm production and sperm morphology. Biol Reprod. 64: 927934. 2001

27. Gill-Sharma MK, Balasinor N, and Parte P. Effect of intermittent treatment with tamoxifen on reproduction in male rats. Asian J Androl. 3: 115-119. 2001. 